\title{
The impact of diabetes on cognitive decline: potential vascular, metabolic, and psychosocial risk factors
}

\author{
Insa Feinkohl ${ }^{*}$, Jackie F. Price ${ }^{1}$, Mark W.J. Strachan ${ }^{2}$ and Brian M. Frier ${ }^{3}$
}

\begin{abstract}
Older people with type 2 diabetes are at increased risk of developing cognitive impairment, for which several potential risk factors have been proposed. The present article reviews evidence in people with type 2 diabetes for associations of cognitive impairment with a range of vascular, metabolic, and psychosocial risk factors, many of which have a higher prevalence in people with type 2 diabetes than in non-diabetic adults of a similar age. Definitive research studies in this field are few in number. The risk factors may be involved in causal pathways or may act as useful markers of cerebrovascular damage (or both), and for which relatively consistent evidence is available, include poor glycemic control, hypoglycemia, microvascular disease, inflammation, and depression. For macrovascular disease, the strength of the association with cognitive impairment appears to depend on which vascular system has been examined. A role for pre-morbid ability in young adulthood as influencing the risk of both diabetes and cognitive impairment has also been suggested. The importance of considering inter-relationships between risk factors when investigating their potential contribution to cognitive impairment in future investigations is discussed.
\end{abstract}

\section{Introduction}

The global pandemic of diabetes is exerting an everincreasing burden on health-care systems. The incidence of dementia is also rising worldwide. Diabetes, which is characterized by chronic hyperglycemia, appears to be associated with an increased risk of developing Alzheimer's disease $(\mathrm{AD})$ and vascular dementia $(\mathrm{VaD})$, both in the general population [1] and in people who have already been diagnosed with a milder form of age-related cognitive impairment (mild cognitive impairment) [2]. With cognitive aging as a continuum, people with type 2 diabetes have been found to experience accelerated cognitive decline within a dementia-free range of between $20 \%$ and $50 \%$ [3], and recent reports have suggested a role of mid-life (rather than late-life) diabetes in particular in promoting this cognitive dysfunction $[4,5]$.

Numerous vascular, metabolic, and psychosocial factors have a potential role in the development of cognitive impairment in populations with diabetes and may contribute to diabetes-related cognitive decline (Fig. 1). Most factors

\footnotetext{
*Correspondence: ifeinkohl@gmail.com

${ }^{1}$ Centre for Population Health Sciences, Medical School, Teviot Place, Edinburgh EH8 9AG, Scotland, UK

Full list of author information is available at the end of the article
}

are inter-related and could influence cognitive ability through a number of different pathophysiological pathways. In this article, we have aimed to provide an overview (rather than a formal systematic review) of the current evidence on risk factors for cognitive impairment in people with diabetes. For that purpose, each risk factor is considered individually and with a focus on prospective epidemiological studies in populations with type 2 diabetes. Where such evidence is lacking, information derived from studies in the general (non-diabetic) population and from adults with type 1 diabetes has been included. It is important to emphasize that even where associations based on observational research are well established, these do not demonstrate causality, and so evaluation of the epidemiological evidence has been supplemented where possible by consideration of intervention studies. Although many of the risk factors are likely to affect cognition through an influence on cerebrovascular disease, on ADtypical pathology or on both, underlying pathophysiological mechanisms are not the main focus of this article, and these have been reviewed extensively elsewhere [6-8]. In addition, possible genetic factors are not addressed. 


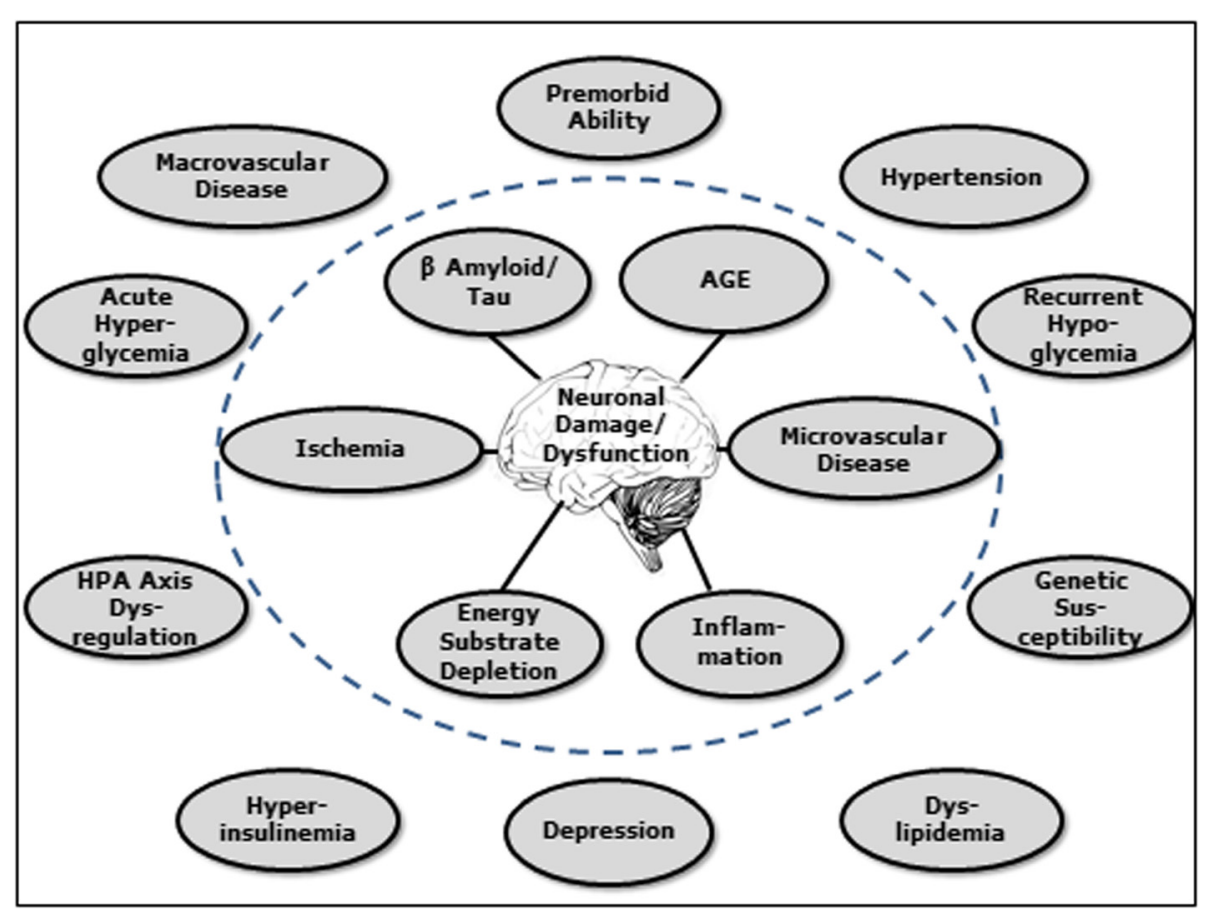

Fig. 1 Potential risk factors contributing to the increased risk of cognitive impairment in older adults with type 2 diabetes. AGE, advanced glycation end-products; HPA, hypothalamic-pituitary axis. Adapted from [72]

The primary studies identified and reviewed in this article are summarized in Tables 1, 2, 3, 4 and are also provided as supplemental data (Additional file 1).

\section{Vascular and metabolic risk factors Dyslipidemia}

Although dyslipidemia is common in type 2 diabetes, few observational studies have examined whether an association exists between plasma lipid concentrations and cognitive function (Table 1). Cognitive function has been reported to be significantly poorer in people with type 2 diabetes who have elevated levels of plasma triglycerides $[9,10]$ and in those with higher cholesterol levels [9], but neither of these observations has been confirmed $[11,12]$. Two investigations even reported protective effects: in one cross-sectional study, dislipidemia was associated with better performance on a task of processing speed [13], and higher total cholesterol was found to decrease the risk of subsequent cognitive impairment short of dementia during an 8-year period in the Fremantle Diabetes Study [14]. However, a small intervention study on the effects of physical exercise on insulin resistance over a period of 12 weeks (which was unsuccessful in inducing a change in insulin resistance) [15] and two further prospective observational studies $[16,17]$ failed to find any association between plasma lipid profiles and subsequent cognitive decline or risk of impairment, with the exception of an apparent association between lower mean high- density lipoprotein during a 6-year period and a steeperthan-expected cognitive decline in a small Japanese study during the same time period [16].

In the Action to Control Cardiovascular Risk in Diabetes-Memory in Diabetes (ACCORD-MIND) randomized controlled trial (RCT), almost 3,000 older people with type 2 diabetes were assigned either to intensive treatment of hyperglycemia or to standard therapy [17]. Around $50 \%$ of participants also entered the only RCT to date to address the effects of a reduction in plasma lipid levels on cognitive decline in people with type 2 diabetes (the other $50 \%$ participated in a trial of anti-hypertensives). Despite a greater reduction in cholesterol levels in patients who received fenofibrate plus simvastatin compared with those receiving placebo plus simvastatin, cognitive function in the two groups declined at similar rates during a 40-month follow-up period [17]. A review of RCTs performed in the general (predominantly nondiabetic) population also concluded that reducing plasma cholesterol does not influence late-life cognitive function [18], consistent with findings from observational studies performed in the general population [2]. The role of dyslipidemia in the development of cognitive impairment in people with diabetes is therefore uncertain.

\section{Hypertension}

Hypertension is common in people with type 2 diabetes and, in general, has received more attention than 
Table 1 Studies of dyslipidemia and cognitive function in type 2 diabetes

Study Sample Design Number Baseline mean Lipids

Lipids Cognitive measures
\begin{tabular}{lll}
\hline Bruce et al. & $\begin{array}{l}\text { Patients with type 2 } \\
\text { [14] (2008) } \\
\text { diabetes participating in } \\
\text { the Fremantle Diabetes }\end{array}$ & $\begin{array}{l}\text { retrospective, } \\
\text { observational }\end{array}$
\end{tabular} Study; Australia

$302 \begin{aligned} & \text { Mean } 76 \pm \\ & 5 \text { years }\end{aligned}$

Total cholesterol

and $\mathrm{HDL}$ at

baseline and

Dementia and MCl identified

from screening instruments/

clinical interview
Adjustment variables

Waist-hip ratio

101 Mean $63 \pm$

8 years

$\mathrm{LDL}$, and $\mathrm{HDL}$

MCl identified on the basis of

sectional

observational

[9] (2011) diabetes; Chin

[11] (2012) diabetes; China

Umegaki Patients with type 2
246 Range 55-74

years

157 Mean $55 \pm \quad$ Triglycerides, MCl identified on the basis of 7 years

2,977 Mean $63 \pm$ 6 years 5 years total cholesterol, cognitive screening instrument $\mathrm{LDL}$, and $\mathrm{HDL}$

Hyperlipidemia Digit Symbol Coding (primary defined as use outcome), MMSE, Rey Auditory of lipid-lowering Verbal Learning, and Stroop medication or (secondary outcomes) untreated LDL

cholesterol total cholesterol and simple reaction time

Mean of HDL and LDL

Composite score from MMSE Digit Symbol Coding, Stroop,
79 Mean $74 \pm$
Chen et al. Patients with type

Cukierman- Patients with type 2

Cross-

sectional

observational

$\begin{array}{ll}\text { diabetes participating in } & \text { sectional } \\ \text { ACCORD-MIND; North } & \text { analysis of }\end{array}$

America

trial on blood

pressure,

lipids, and

glycemic

contro

Perlmutter Patients with type $2 \quad$ Cross-

et al. [10] diabetes: USA

(1988)

sectional,

Six-year

et al. [16] diabetes; Japan

(2014) prospective,

observational
Triglycerides and Digit Symbol Coding, Digit Span, measured at baseline and annual followups and word recall. Analyses of on the basis of composite score

40-month

Williamson Patients with type 2

et al. [17] diabetes participating in

trial on blood

arm, receiving

pressure,
1,538 Mean $62 \pm$

6 years
Successful

manipulation of Total brain volume at baseline cholesterol Coding (primary Digit Symbo levels (groups
MMSE, Rey Auditory Verba 'decliners' versus 'non-decliners' and individual cognitive tests.

None

Age

one

Age

with better performance on Digit Symbol Coding. No findings for other cognitive tests.

Plasma glucose, $\mathrm{HbA1c}$, body mass index, and history of hypertension

Age, education, estimated glomerular filtration rate, renin-angiotensin system inhibitor use, paraventricular hyperintensities, and deep white matter hyperintensities
Glycemia treatment arm, visit effect, clinical center, and history of cardiovascular disease
Association with cognitive

No associations in unadjusted

analyses. Higher total cholesterol 8 years earlier protective of cognitive impairment short of dementia impairment) at baseline (finding independent of waist-hip ratio)

Higher triglycerides, total

cholesterol, and LDL in MCI of $\mathrm{MCl}$. Negative correlation of total cholesterol with scores on cognitive screening instrument in patients with $\mathrm{MCl}$. No finding for HDL

No association
Lower cognitive function in 'high' compared with 'low' triglyceride groups (fully adjusted analysis); association of cholesterol with triglyceride (unadjusted analysis).

Lower mean 6-year HDL in 'decliners' compared with 'nondecliners' on composite score and Stroop (unadjusted analysis). No finding for LDL. Mean 6-year $\mathrm{HDL}$ significant predictor in model of risk of decline on com posite score (fully adjusted analysis).

No difference in 20- or 40-month cognitive decline or 40-month change in total brain volume 
Table 1 Studies of dyslipidemia and cognitive function in type 2 diabetes (Continued)

\begin{tabular}{|c|c|c|c|c|c|c|c|c|}
\hline & $\begin{array}{l}\text { simvastatin + fenofibrate } \\
\text { or simvastatin + placebo; } \\
\text { North America }\end{array}$ & $\begin{array}{l}\text { glycemic } \\
\text { control }\end{array}$ & & & $\begin{array}{l}\text { differed on } \\
\text { cholesterol } \\
\text { following } \\
\text { intervention). }\end{array}$ & $\begin{array}{l}\text { Learning, and Stroop (secondary } \\
\text { outcomes) at baseline and } 20 \\
\text { and } 40 \text { months }\end{array}$ & & $\begin{array}{l}\text { between intervention and control } \\
\text { groups of lipid trial }\end{array}$ \\
\hline $\begin{array}{l}\text { Van Harten } \\
\text { et al. [12] } \\
\text { (2007) }\end{array}$ & $\begin{array}{l}\text { Patients with type } 2 \\
\text { diabetes; The } \\
\text { Netherlands }\end{array}$ & $\begin{array}{l}\text { Cross- } \\
\text { sectional, } \\
\text { observational }\end{array}$ & 92 & $\begin{array}{l}\text { Mean } 73 \pm \\
6 \text { years }\end{array}$ & $\begin{array}{l}\text { Total } \\
\text { cholesterol/HDL } \\
\text { ratio }\end{array}$ & $\begin{array}{l}\text { Cognitive screening instruments, } \\
\text { composite scores of four } \\
\text { cognitive domains derived from } \\
\text { scores on battery of } 10 \text { cognitive } \\
\text { tests }\end{array}$ & $\begin{array}{l}\text { Duration of diabetes, } \\
\text { HbA1c, insulin use, } \\
\text { hypertension, and } \\
\text { polyneuropathy }\end{array}$ & No association \\
\hline $\begin{array}{l}\text { Yanagawa } \\
\text { et al. [15] } \\
(2011)\end{array}$ & $\begin{array}{l}\text { Patients with diabetes } \\
\text { receiving exercise } \\
\text { program four times per } \\
\text { week versus none; } \\
\text { Japan }\end{array}$ & $\begin{array}{l}\text { 12-week trial } \\
\text { on physical } \\
\text { exercise } \\
\text { intervention }\end{array}$ & 16 & $\begin{array}{l}\text { Mean } 71 \pm \\
4 \text { years }\end{array}$ & $\begin{array}{l}\mathrm{HDL} \text { and } \mathrm{LDL} \\
\text { (no intervention } \\
\text { effect on } \mathrm{HDL} \\
\text { and } \mathrm{LDL} \text { ) }\end{array}$ & $\begin{array}{l}\text { MMSE, word recall, Digit Symbol } \\
\text { Coding, Stroop, and Trail-Making } \\
\text { Test }\end{array}$ & $\begin{array}{l}\text { Age, education, and body } \\
\text { mass index }\end{array}$ & $\begin{array}{l}\text { No difference in cognitive } \\
\text { function between treatment } \\
\text { groups following intervention }\end{array}$ \\
\hline
\end{tabular}

ACCORD-MIND, Action to Control Cardiovascular Risk in Diabetes-Memory in Diabetes; HDL, high-density lipoprotein; LDL, low-density lipoprotein; MCI, mild cognitive impairment; MMSE, Mini-Mental State Examination 
Table 2 Studies of hypertension and cognitive function in type 2 diabetes

\begin{tabular}{|c|c|c|c|c|c|c|c|c|}
\hline Study & Sample & Design & Number & $\begin{array}{l}\text { Baseline mean } \\
\text { age }\end{array}$ & Blood pressure & Cognitive measures & $\begin{array}{l}\text { Adjustment } \\
\text { variables }\end{array}$ & $\begin{array}{l}\text { Association with cognitive } \\
\text { function }\end{array}$ \\
\hline $\begin{array}{l}\text { Chen et al. } \\
\text { [11] (2012) }\end{array}$ & $\begin{array}{l}\text { Patients with type } 2 \\
\text { diabetes; China }\end{array}$ & $\begin{array}{l}\text { Cross- } \\
\text { sectional, } \\
\text { observational }\end{array}$ & 157 & $\begin{array}{l}\text { Mean } 55 \pm \\
7 \text { years }\end{array}$ & $\begin{array}{l}\text { Hypertension defined on } \\
\text { the basis of systolic blood } \\
\text { pressure and diastolic } \\
\text { blood pressure }\end{array}$ & $\begin{array}{l}\mathrm{MCl} \text { identified on the basis of } \\
\text { cognitive screening instrument }\end{array}$ & None & $\begin{array}{l}\text { Higher prevalence of hypertension } \\
\text { in group with MCl compared with } \\
\text { group free of MCl. Negative } \\
\text { correlation of presence with } \\
\text { hypertension with cognitive scores. } \\
\text { No findings for blood pressure as } \\
\text { continuous measure. }\end{array}$ \\
\hline $\begin{array}{l}\text { Bruce et al. } \\
\text { [14] (2008) }\end{array}$ & $\begin{array}{l}\text { Patients with type } 2 \\
\text { diabetes } \\
\text { participating in the } \\
\text { Fremantle Diabetes } \\
\text { Study; Australia }\end{array}$ & $\begin{array}{l}\text { 8-year } \\
\text { retrospective, } \\
\text { observational }\end{array}$ & 302 & $\begin{array}{l}\text { Mean } 76 \pm \\
5 \text { years }\end{array}$ & $\begin{array}{l}\text { Systolic blood pressure and } \\
\text { diastolic blood pressure at } \\
\text { baseline and } 8 \text { years earlier }\end{array}$ & $\begin{array}{l}\text { Dementia and MCI identified from } \\
\text { screening instruments/clinical } \\
\text { interview }\end{array}$ & $\begin{array}{l}\text { Age and } \\
\text { duration of } \\
\text { diabetes }\end{array}$ & $\begin{array}{l}\text { Prospective analyses: higher } \\
\text { diastolic blood pressure } 8 \text { years } \\
\text { earlier associated with increased } \\
\text { risk of } \mathrm{AD} \text { (but not } \mathrm{MCl} \text { or any } \\
\text { dementia) at follow-up. No findings } \\
\text { in cross-sectional analyses. }\end{array}$ \\
\hline $\begin{array}{l}\text { Bruce et al. } \\
\text { [21] (2008) }\end{array}$ & $\begin{array}{l}\text { Patients with type } 2 \\
\text { diabetes } \\
\text { participating in the } \\
\text { Fremantle Diabetes } \\
\text { Study; Australia }\end{array}$ & $\begin{array}{l}\text { 8-year } \\
\text { retrospective, } \\
\text { 2-year } \\
\text { prospective, } \\
\text { observational }\end{array}$ & 205 & $\begin{array}{l}\text { Mean } 75 \pm \\
4 \text { years }\end{array}$ & $\begin{array}{l}\text { Systolic blood pressure and } \\
\text { diastolic blood pressure } \\
\text { measured } 8 \text { years prior to } \\
\text { baseline cognitive } \\
\text { assessment }\end{array}$ & $\begin{array}{l}\text { Dementia and } \mathrm{MCl} \text { identified from } \\
\text { screening instruments/clinical } \\
\text { interview at baseline and 2-year } \\
\text { follow-up. 'Cognitive } \\
\text { decline' defined as downward con- } \\
\text { version between 'normal', MCl, and } \\
\text { dementia. }\end{array}$ & None & No association \\
\hline $\begin{array}{l}\text { Cukierman- } \\
\text { Yaffe et al. } \\
\text { [13] (2009) }\end{array}$ & $\begin{array}{l}\text { Patients with type } 2 \\
\text { diabetes } \\
\text { participating in } \\
\text { ACCORD-MIND; } \\
\text { North America }\end{array}$ & $\begin{array}{l}\text { Cross-sectional } \\
\text { analysis of trial } \\
\text { on blood } \\
\text { pressure, lipids, } \\
\text { and glycemic } \\
\text { control }\end{array}$ & 2,977 & $\begin{array}{l}\text { Mean } 63 \pm \\
6 \text { years }\end{array}$ & $\begin{array}{l}\text { Hypertension defined as } \\
\text { use of anti-hypertensive } \\
\text { medication or self-report of } \\
\text { hypertension }\end{array}$ & $\begin{array}{l}\text { Digit Symbol Coding (primary } \\
\text { outcome), MMSE, Rey Auditory } \\
\text { Verbal Learning, and Stroop } \\
\text { (secondary outcomes) }\end{array}$ & Age & $\begin{array}{l}\text { Association of hypertension with } \\
\text { poorer performance on Digit } \\
\text { Symbol Coding. No findings for } \\
\text { other cognitive tests. }\end{array}$ \\
\hline \multirow[t]{2}{*}{$\begin{array}{l}\text { Hassing } \\
\text { et al. [19] } \\
(2004)\end{array}$} & \multirow{2}{*}{$\begin{array}{l}\text { OCTO-Twin Study of } \\
\text { people without } \\
\text { diabetes/ } \\
\text { hypertension, } \\
\text { diabetes or } \\
\text { hypertension alone, } \\
\text { or co-morbid dia- } \\
\text { betes/ } \\
\text { hypertension; } \\
\text { Sweden }\end{array}$} & \multirow[t]{2}{*}{$\begin{array}{l}\text { 6-year } \\
\text { prospective, } \\
\text { observational }\end{array}$} & \multirow[t]{2}{*}{258} & \multirow[t]{2}{*}{$\begin{array}{l}\text { Mean } 83 \pm \\
2 \text { years }\end{array}$} & \multirow{2}{*}{$\begin{array}{l}\text { Hypertension defined as } \\
\text { use of anti-hypertensive } \\
\text { medication or on the basis } \\
\text { of systolic blood pressure } \\
\text { and diastolic blood pressure } \\
\text { from medical records }\end{array}$} & \multirow{2}{*}{$\begin{array}{l}\text { MMSE administered at baseline and } \\
\text { at 2-year intervals, dementia diag- } \\
\text { nosis prevalent at baseline, and in- } \\
\text { cident dementia diagnosis }\end{array}$} & \multirow[t]{2}{*}{$\begin{array}{l}\text { Age, sex, } \\
\text { education, } \\
\text { smoking, } \\
\text { angina, Ml, CHF, } \\
\text { stroke, and TIA }\end{array}$} & $\begin{array}{l}\text { Co-morbid diabetes/hypertension } \\
\text { associated with steeper decline on } \\
\text { MMSE (compared with group free } \\
\text { of both conditions). }\end{array}$ \\
\hline & & & & & & & & $\begin{array}{l}\text { Statistically non-significant trend for } \\
\text { higher prevalence and } \\
\text { incidence of dementia in } \\
\text { co-morbid } \\
\text { diabetes/hypertension group } \\
\text { than in remaining groups. }\end{array}$ \\
\hline \multirow[t]{2}{*}{$\begin{array}{l}\text { Johnson } \\
\text { et al. [22] } \\
\text { (2012) }\end{array}$} & \multirow[t]{2}{*}{$\begin{array}{l}\text { National cohort of } \\
\text { veterans with } \\
\text { diabetes; USA }\end{array}$} & \multirow{2}{*}{$\begin{array}{l}\text { 2-year } \\
\text { retrospective, } \\
\text { observational } \\
\text { study of } \\
\text { hospital } \\
\text { records }\end{array}$} & \multirow[t]{2}{*}{377,838} & \multirow[t]{2}{*}{$\begin{array}{l}\text { Mean } 76 \pm \\
6 \text { years }\end{array}$} & \multirow[t]{2}{*}{$\begin{array}{l}\text { ICD codes for hypertension } \\
\text { at baseline ( } 2 \text { years before } \\
\text { analysis of incident } \\
\text { dementia) }\end{array}$} & \multirow[t]{2}{*}{$\begin{array}{l}\text { ICD codes for incident dementia } \\
\text { diagnosis }\end{array}$} & \multirow{2}{*}{$\begin{array}{l}\text { Age, ethnicity, } \\
\text { geographic } \\
\text { area, duration } \\
\text { of diabetes, } \\
\text { co-morbidity } \\
\text { according to } \\
\text { HCC scores, } \\
\text { and medication } \\
\text { use }\end{array}$} & $\begin{array}{l}8 \% \text { increased risk of developing } \\
\text { dementia during follow-up in } \\
\text { patients with co-morbid } \\
\text { hypertension at baseline. }\end{array}$ \\
\hline & & & & & & & & $\begin{array}{l}\text { Decreased risk in patients on anti- } \\
\text { hypertensive medication (effect } \\
\text { size dependent on } \\
\text { medication), except for } \\
\text { increased risk in patients receiving } \\
\text { a-adrenoceptor blockers. }\end{array}$ \\
\hline
\end{tabular}


Table 2 Studies of hypertension and cognitive function in type 2 diabetes (Continued)

\begin{tabular}{|c|c|c|c|c|c|c|c|c|}
\hline $\begin{array}{l}\text { Manschot } \\
\text { et al. [20] } \\
\text { (2006) }\end{array}$ & $\begin{array}{l}\text { Patients with type } 2 \\
\text { diabetes } \\
\text { participating in the } \\
\text { Utrecht Diabetic } \\
\text { Encephalopathy } \\
\text { Study; The } \\
\text { Netherlands }\end{array}$ & $\begin{array}{l}\text { Cross- } \\
\text { sectional, } \\
\text { observational }\end{array}$ & 122 & $\begin{array}{l}\text { Mean } 66 \pm \\
6 \text { years }\end{array}$ & $\begin{array}{l}\text { Hypertension defined on } \\
\text { the basis of systolic blood } \\
\text { pressure and diastolic } \\
\text { blood pressure or use of } \\
\text { anti-hypertensive } \\
\text { medication }\end{array}$ & $\begin{array}{l}\text { Composite scores on five cognitive } \\
\text { domains from } 11 \text { cognitive tests, } \\
\text { estimate of pre-morbid ability, cor- } \\
\text { tical atrophy, and white matter } \\
\text { lesions }\end{array}$ & $\begin{array}{l}\text { Age, sex, and } \\
\text { estimated pre- } \\
\text { morbid ability }\end{array}$ & $\begin{array}{l}\text { Statistically non-significant trend } \\
\text { for lower scores on all cognitive } \\
\text { domains except memory in } \\
\text { patients with hypertension. Higher } \\
\text { blood pressure } \\
\text { associated with higher scores on } \\
\text { memory domain and with greater } \\
\text { severity of white matter lesions. }\end{array}$ \\
\hline $\begin{array}{l}\text { Manschot } \\
\text { et al. [25] } \\
\text { (2007) }\end{array}$ & $\begin{array}{l}\text { Patients with type } 2 \\
\text { diabetes } \\
\text { participating in the } \\
\text { Utrecht Diabetic } \\
\text { Encephalopathy } \\
\text { Study; The } \\
\text { Netherlands }\end{array}$ & $\begin{array}{l}\text { Cross- } \\
\text { sectional, } \\
\text { observational }\end{array}$ & 122 & $\begin{array}{l}\text { Mean } 66 \pm \\
6 \text { years }\end{array}$ & $\begin{array}{l}\text { Hypertension defined on } \\
\text { the basis of systolic blood } \\
\text { pressure and diastolic } \\
\text { blood pressure or use of } \\
\text { anti-hypertensive } \\
\text { medication }\end{array}$ & $\begin{array}{l}\text { Composite score from } 11 \text { cognitive } \\
\text { tests, estimate of pre-morbid ability, } \\
\text { cortical atrophy, and white matter } \\
\text { lesions }\end{array}$ & $\begin{array}{l}\text { Age, sex, and } \\
\text { estimated pre- } \\
\text { morbid ability }\end{array}$ & $\begin{array}{l}\text { Statistically non-significant trend } \\
\text { for lower cognitive function in pa- } \\
\text { tients with hypertension (reaches } \\
\text { statistical significance in final } \\
\text { model including age, } \\
\text { estimated pre-morbid ability, } \\
\text { lipid-lowering drugs, and history of } \\
\text { any vascular event). Higher blood } \\
\text { pressure associated with greater se- } \\
\text { verity of white matter lesions. }\end{array}$ \\
\hline $\begin{array}{l}\text { Umegaki } \\
\text { et al. [16] } \\
\text { (2014) }\end{array}$ & $\begin{array}{l}\text { Patients with type } 2 \\
\text { diabetes; Japan }\end{array}$ & $\begin{array}{l}\text { 6-year } \\
\text { prospective, } \\
\text { observational }\end{array}$ & 79 & $\begin{array}{l}\text { Mean } 74 \pm \\
5 \text { years }\end{array}$ & $\begin{array}{l}\text { Mean of systolic blood } \\
\text { pressure and diastolic } \\
\text { blood pressure measured at } \\
\text { baseline and annual follow- } \\
\text { ups }\end{array}$ & $\begin{array}{l}\text { Composite score from MMSE, Digit } \\
\text { Symbol Coding, Stroop, and word } \\
\text { recall. Analyses of 'decliners' versus } \\
\text { 'non-decliners' on bases of } \\
\text { composite score and individual } \\
\text { cognitive tests. }\end{array}$ & None & No association \\
\hline $\begin{array}{l}\text { Williamson } \\
\text { et al. [17] } \\
\text { (2014) }\end{array}$ & $\begin{array}{l}\text { Patients with type } 2 \\
\text { diabetes } \\
\text { participating in } \\
\text { ACCORD-MIND } \\
\text { blood } \\
\text { pressure arm, with } \\
\text { systolic blood pres- } \\
\text { sure goal of } 120 \text { ver- } \\
\text { sus } 140 \text { mmHg; } \\
\text { North America }\end{array}$ & $\begin{array}{l}\text { 40-month } \\
\text { trial on blood } \\
\text { pressure, } \\
\text { lipids, and } \\
\text { glycemic } \\
\text { control }\end{array}$ & 1,439 & $\begin{array}{l}\text { Mean } 62 \pm \\
6 \text { years }\end{array}$ & $\begin{array}{l}\text { Successful manipulation of } \\
\text { blood pressure (groups } \\
\text { differed on blood pressure } \\
\text { following intervention) }\end{array}$ & $\begin{array}{l}\text { Total brain volume at baseline and } \\
40 \text { months, Digit Symbol Coding } \\
\text { (primary outcome), MMSE, Rey } \\
\text { Auditory Verbal Learning, and } \\
\text { Stroop (secondary outcomes) at } \\
\text { baseline and } 20 \text { and } 40 \text { months }\end{array}$ & $\begin{array}{l}\text { Glycemia } \\
\text { treatment arm, } \\
\text { visit effect, } \\
\text { clinical center, } \\
\text { and history of } \\
\text { cardiovascular } \\
\text { disease }\end{array}$ & $\begin{array}{l}\text { No difference in 20- or 40-month } \\
\text { cognitive decline treatment and } \\
\text { control groups. Greater reduction } \\
\text { in total brain volume in } \\
\text { intervention than in control group. }\end{array}$ \\
\hline
\end{tabular}

ACCORD-MIND, Action to Control Cardiovascular Risk in Diabetes-Memory in Diabetes; AD, Alzheimer's dementia; CHF, congestive heart failure; HCC, Centers for Medicare and Medicaid Services' Hierarchical Condition Categories; ICD, International Classification of Diseases; MCI, mild cognitive impairment; MI, myocardial infarction; MMSE, Mini-Mental State Examination; OCTO-Twin Study, Origin of Variance in the Old Old Study: Octogenarian Twins; TIA, transient ischemic attack 
Table 3 Studies of hyperglycemia or hyperinsulinemia and cognitive function in type 2 diabetes

\begin{tabular}{|c|c|c|c|c|c|c|c|c|}
\hline Study & Sample & Design & Number & $\begin{array}{l}\text { Baseline mean } \\
\text { age }\end{array}$ & $\begin{array}{l}\text { Hyperglycemia/ } \\
\text { hyperinsulinemia }\end{array}$ & Cognitive measures & Adjustment variables & $\begin{array}{l}\text { Association with cognitive } \\
\text { function }\end{array}$ \\
\hline $\begin{array}{l}\text { Abbatecola } \\
\text { et al. [30] } \\
(2006)\end{array}$ & $\begin{array}{l}\text { Patients with } \\
\text { diabetes free of } \\
\text { vascular disease, } \\
\text { receiving } \\
\text { repaglinide or } \\
\text { glibenclamide; Italy }\end{array}$ & $\begin{array}{l}12 \text {-month } \\
\text { trial on } \\
\text { glycemic } \\
\text { control }\end{array}$ & 156 & $\begin{array}{l}\text { Mean } 74 \pm \\
2 \text { years }\end{array}$ & $\begin{array}{l}\text { Variation in post-prandial } \\
\text { blood glucose, fasting plasma } \\
\text { glucose, and HbA1c. (No dif- } \\
\text { ference between groups in } \\
\text { reduction of HbA1c and } \\
\text { plasma glucose during trial. } \\
\text { Decline in variation in post- } \\
\text { prandial glucose only in group } \\
\text { treated with repaglinide.) }\end{array}$ & $\begin{array}{l}\text { Composite score of attention/ } \\
\text { executive function (Trail-Making } \\
\text { Test, Digit Span, and verbal } \\
\text { fluency), MMSE, cortical atrophy, } \\
\text { and white matter lesions }\end{array}$ & $\begin{array}{l}\text { Age, education, } \\
\text { physical activity, } \\
\text { depression, blood } \\
\text { pressure, cIMT, insulin } \\
\text { resistance, and body } \\
\text { mass index }\end{array}$ & $\begin{array}{l}\text { Association of higher variation } \\
\text { in fasting plasma glucose and } \\
\text { post-prandial blood glucose } \\
\text { with lower cognitive function } \\
\text { across groups at baseline } \\
\text { (fully adjusted analyses). } \\
\text { Composite score and MMSE } \\
\text { declined in glibenclamide } \\
\text { but not in repaglinide group } \\
\text { during trial (analyses controlling } \\
\text { only for HbA1c and variation in } \\
\text { fasting plasma glucose). }\end{array}$ \\
\hline $\begin{array}{l}\text { Bruce et al. } \\
\text { [14] (2008) }\end{array}$ & $\begin{array}{l}\text { Patients with type } 2 \\
\text { diabetes } \\
\text { participating in the } \\
\text { Fremantle Diabetes } \\
\text { Study; Australia }\end{array}$ & $\begin{array}{l}\text { 8-year } \\
\text { retrospective, } \\
\text { observational }\end{array}$ & 302 & $\begin{array}{l}\text { Mean } 76 \pm \\
5 \text { years }\end{array}$ & $\begin{array}{l}\text { HbA1c at baseline and } 8 \text { years } \\
\text { earlier }\end{array}$ & $\begin{array}{l}\text { Dementia and MCl identified } \\
\text { from screening instruments/ } \\
\text { clinical interview }\end{array}$ & None & No association \\
\hline $\begin{array}{l}\text { Bruce et al. } \\
{[21](2008)}\end{array}$ & $\begin{array}{l}\text { Patients with type } 2 \\
\text { diabetes } \\
\text { participating in the } \\
\text { Fremantle Diabetes } \\
\text { Study; Australia }\end{array}$ & $\begin{array}{l}\text { 8-year } \\
\text { retrospective, } \\
\text { 2-year pro- } \\
\text { spective, } \\
\text { observational. }\end{array}$ & 205 & $\begin{array}{l}\text { Mean } 75 \pm \\
4 \text { years }\end{array}$ & $\begin{array}{l}\text { HbA1c } 8 \text { years prior to } \\
\text { baseline cognitive assessment }\end{array}$ & $\begin{array}{l}\text { Dementia and } \mathrm{MCl} \text { identified } \\
\text { from screening instruments/ } \\
\text { clinical interview at baseline } \\
\text { and at 2-year follow-up. 'Cog- } \\
\text { nitive decline' defined as } \\
\text { downward conversion between } \\
\text { 'normal', MCl, and dementia. }\end{array}$ & None & No association \\
\hline $\begin{array}{l}\text { Chen et al. } \\
\text { [9] (2011) }\end{array}$ & $\begin{array}{l}\text { Patients with type } 2 \\
\text { diabetes; China }\end{array}$ & $\begin{array}{l}\text { Cross- } \\
\text { sectional, } \\
\text { observational }\end{array}$ & 101 & $\begin{array}{l}\text { Mean } 63 \pm \\
8 \text { years }\end{array}$ & $\mathrm{HbA1c}$ & $\begin{array}{l}\mathrm{MCl} \text { identified on the basis of } \\
\text { cognitive screening } \\
\text { instrument }\end{array}$ & None & $\begin{array}{l}\text { Higher } \mathrm{HbA} 1 \mathrm{c} \text { in group with } \\
\mathrm{MCl} \text { compared with group } \\
\text { free of } \mathrm{MCl}\end{array}$ \\
\hline $\begin{array}{l}\text { Chen et al. } \\
\text { [11] (2012) }\end{array}$ & $\begin{array}{l}\text { Patients with type } 2 \\
\text { diabetes; China }\end{array}$ & $\begin{array}{l}\text { Cross- } \\
\text { sectional, } \\
\text { observational }\end{array}$ & 157 & $\begin{array}{l}\text { Mean } 55 \pm \\
7 \text { years }\end{array}$ & $\mathrm{HbA1c}$ & $\begin{array}{l}\mathrm{MCl} \text { identified on the basis of } \\
\text { cognitive screening } \\
\text { instrument }\end{array}$ & None & No association \\
\hline $\begin{array}{l}\text { Cukierman- } \\
\text { Yaffe et al. } \\
\text { [13] (2009) }\end{array}$ & $\begin{array}{l}\text { Patients with type } 2 \\
\text { diabetes } \\
\text { participating in } \\
\text { ACCORD-MIND; } \\
\text { North America }\end{array}$ & $\begin{array}{l}\text { Cross- } \\
\text { sectional } \\
\text { analysis of } \\
\text { trial on blood } \\
\text { pressure, } \\
\text { lipids, and } \\
\text { glycemic } \\
\text { control }\end{array}$ & 2,977 & $\begin{array}{l}\text { Mean } 63 \pm \\
6 \text { years }\end{array}$ & $\begin{array}{l}\text { HbA1c and fasting plasma } \\
\text { glucose }\end{array}$ & $\begin{array}{l}\text { Digit Symbol Coding (primary } \\
\text { outcome), MMSE, Rey } \\
\text { Auditory Verbal Learning, and } \\
\text { Stroop (secondary outcomes) }\end{array}$ & $\begin{array}{l}\text { Total of } 18 \\
\text { demographic and } \\
\text { clinical risk factors }\end{array}$ & $\begin{array}{l}\text { Higher HbA1c associated with } \\
\text { lower Digit Symbol Coding. } \\
\text { Findings for other cognitive } \\
\text { tests did not survive full } \\
\text { adjustment. No findings for } \\
\text { fasting plasma glucose. }\end{array}$ \\
\hline $\begin{array}{l}\text { Launer } \\
\text { et al. [28] } \\
\text { (2011) }\end{array}$ & $\begin{array}{l}\text { Patients with type } 2 \\
\text { diabetes } \\
\text { participating in } \\
\text { ACCORD-MIND trial, } \\
\text { with HbA1c targets } \\
\text { of }<6.0 \% \text { versus } \\
7.0 \% \text { to } 7.9 \% \text {; } \\
\text { North America }\end{array}$ & $\begin{array}{l}\text { 40-month } \\
\text { trial on blood } \\
\text { pressure, } \\
\text { lipids and } \\
\text { glycemic } \\
\text { control }\end{array}$ & 2,977 & $\begin{array}{l}\text { Mean } 62 \pm \\
6 \text { years }\end{array}$ & $\begin{array}{l}\text { Successful manipulation of } \\
\text { glycemic control. (Treatment } \\
\text { groups differed in glycemic } \\
\text { control following } \\
\text { intervention.) }\end{array}$ & $\begin{array}{l}\text { Total brain volume and } \\
\text { abnormal white matter at } \\
\text { baseline and } 40 \text { months. Digit } \\
\text { Symbol Coding (primary } \\
\text { outcome), MMSE, Rey } \\
\text { Auditory Verbal Learning, and } \\
\text { Stroop (secondary outcomes) } \\
\text { at baseline and } 20 \text { and } \\
40 \text { months. }\end{array}$ & $\begin{array}{l}\text { Second trial } \\
\text { assignment (lipid or } \\
\text { blood pressure trials), } \\
\text { group allocation within } \\
\text { second trial } \\
\text { assignment, clinical } \\
\text { center, and history of } \\
\text { cardiovascular disease }\end{array}$ & $\begin{array}{l}\text { No difference in 20- or 40- } \\
\text { month cognitive decline be- } \\
\text { tween intervention groups (fully } \\
\text { adjusted analyses). Total brain } \\
\text { volume declined at slower rate } \\
\text { in intensively treated compared } \\
\text { with standard treatment groups } \\
\text { (independent of adjustment } \\
\text { variables and of age, sex, }\end{array}$ \\
\hline
\end{tabular}


Table 3 Studies of hyperglycemia or hyperinsulinemia and cognitive function in type 2 diabetes (Continued)

\begin{tabular}{|c|c|c|c|c|c|c|c|c|}
\hline $\begin{array}{l}\text { Manschot } \\
\text { et al. [20] } \\
\text { (2006) }\end{array}$ & $\begin{array}{l}\text { Patients with type } 2 \\
\text { diabetes } \\
\text { participating in the } \\
\text { Utrecht Diabetic } \\
\text { Encephalopathy } \\
\text { Study; The } \\
\text { Netherlands }\end{array}$ & $\begin{array}{l}\text { Cross- } \\
\text { sectional, } \\
\text { observational }\end{array}$ & 122 & $\begin{array}{l}\text { Mean } 66 \pm \\
6 \text { years }\end{array}$ & $\mathrm{HbA} 1 \mathrm{c}$ & $\begin{array}{l}\text { Composite scores on five } \\
\text { cognitive domains from } 11 \\
\text { cognitive tests, estimate of } \\
\text { pre-morbid ability, cortical } \\
\text { atrophy, and white matter } \\
\text { lesions }\end{array}$ & $\begin{array}{l}\text { Age, sex, and } \\
\text { estimated pre-morbid } \\
\text { ability }\end{array}$ & $\begin{array}{l}\text { Association of higher } \mathrm{HbA1c} \\
\text { with steeper estimated } \\
\text { lifetime decline in processing } \\
\text { speed. No association of } \\
\text { HbA1c with brain imaging } \\
\text { data. }\end{array}$ \\
\hline $\begin{array}{l}\text { Manschot } \\
\text { et al. [25] } \\
(2007)\end{array}$ & $\begin{array}{l}\text { Patients with type } 2 \\
\text { diabetes } \\
\text { participating in the } \\
\text { Utrecht Diabetic } \\
\text { Encephalopathy } \\
\text { Study; The } \\
\text { Netherlands }\end{array}$ & $\begin{array}{l}\text { Cross- } \\
\text { sectional, } \\
\text { observational }\end{array}$ & 122 & $\begin{array}{l}\text { Mean } 66 \pm \\
6 \text { years }\end{array}$ & $\mathrm{HbA1c}$ and plasma insulin & $\begin{array}{l}\text { Composite score from } 11 \\
\text { cognitive tests, estimate of } \\
\text { pre-morbid ability, cortical } \\
\text { atrophy, and white matter } \\
\text { lesions }\end{array}$ & $\begin{array}{l}\text { Age, sex, and } \\
\text { estimated pre-morbid } \\
\text { ability }\end{array}$ & $\begin{array}{l}\text { Association of higher } \mathrm{HbA} 1 \mathrm{c} \\
\text { with steeper estimated } \\
\text { lifetime decline in overall } \\
\text { cognitive function. } \\
\text { Association of higher insulin } \\
\text { with greater severity of white } \\
\text { matter lesions. }\end{array}$ \\
\hline $\begin{array}{l}\text { Ravona- } \\
\text { Springer } \\
\text { et al. [27] } \\
\text { (2014) }\end{array}$ & $\begin{array}{l}\text { Patients with type } 2 \\
\text { diabetes } \\
\text { participating in the } \\
\text { Israel Diabetes and } \\
\text { Cognitive Decline } \\
\text { Study; Israel }\end{array}$ & $\begin{array}{l}12 \text {-year } \\
\text { retrospective } \\
\text { observational }\end{array}$ & 835 & $\begin{array}{l}\text { Mean } 73 \pm \\
5 \text { years }\end{array}$ & $\begin{array}{l}\text { Data on HbA1c from diabetes } \\
\text { register (mean } 18 \pm 10 \\
\text { measurements per patient). } \\
\text { Six HbA1c trajectories } \\
\text { identified (for example, high/ } \\
\text { increasing and high/stable). }\end{array}$ & $\begin{array}{l}\text { Measured at } 12 \text { years only: } \\
\text { CDR, MMSE, and battery of } \\
\text { seven cognitive tests. Sum of } \\
\text { z-scores calculated for four } \\
\text { cognitive domains. }\end{array}$ & $\begin{array}{l}\text { Age, sex, education, } \\
\text { cardiovascular disease, } \\
\text { years in diabetes } \\
\text { register, anti-diabetes } \\
\text { treatment, and } \\
\text { depression }\end{array}$ & $\begin{array}{l}\text { Associations of HbA1c } \\
\text { trajectories with level of } \\
\text { overall cognitive function, } \\
\text { semantic categorization, and } \\
\text { executive function. Relatively } \\
\text { poorest cognitive function in } \\
\text { high/decreasing group and } \\
\text { high/increasing groups. } \\
\text { Relatively highest } \\
\text { performance in low/stable } \\
\text { group. }\end{array}$ \\
\hline $\begin{array}{l}\text { Ryan et al. } \\
\text { [29] (2006) }\end{array}$ & $\begin{array}{l}\text { Patients with type } 2 \\
\text { diabetes, receiving } \\
\text { rosiglitazone or } \\
\text { glibenclamide; USA }\end{array}$ & 24-week trial & 145 & $\begin{array}{l}\text { Mean } 60 \pm \\
1 \text { years }\end{array}$ & $\begin{array}{l}\text { Successful manipulation of } \\
\text { glycemic control and insulin } \\
\text { sensitivity. (Treatment groups } \\
\text { differed in fasting plasma } \\
\text { glucose and fasting serum } \\
\text { insulin following intervention.) }\end{array}$ & $\begin{array}{l}\text { CANTAB, Digit Symbol } \\
\text { Coding, Rey Auditory Verbal } \\
\text { Learning, and estimate of } \\
\text { pre-morbid ability }\end{array}$ & $\begin{array}{l}\text { Age, center, pre- } \\
\text { morbid ability, and } \\
\text { baseline measurement } \\
\text { of fasting plasma glu- } \\
\text { cose/insulin }\end{array}$ & $\begin{array}{l}\text { Cognitive function improved } \\
\text { equally in both treatment } \\
\text { groups (fully adjusted } \\
\text { analysis). Correlation of } \\
\text { reduction in fasting plasma } \\
\text { glucose with improvements } \\
\text { in working memory across } \\
\text { groups (unadjusted analysis). } \\
\text { No finding for insulin. }\end{array}$ \\
\hline $\begin{array}{l}\text { Seaquist } \\
\text { et al. [41] } \\
\text { (2013) }\end{array}$ & $\begin{array}{l}\text { Patients with type } 2 \\
\text { diabetes } \\
\text { participating in } \\
\text { ACCORD-MIND, with } \\
\text { HbA1c targets of } \\
\text { less than } 6.0 \% \text { ver- } \\
\text { sus } 7.0 \% \text { to } 7.9 \% \text {; } \\
\text { North America }\end{array}$ & $\begin{array}{l}\text { 40-month } \\
\text { trial on blood } \\
\text { pressure, } \\
\text { lipids, and } \\
\text { glycemic } \\
\text { control }\end{array}$ & 2,977 & $\begin{array}{l}\text { Mean } 62 \pm \\
6 \text { years }\end{array}$ & $\begin{array}{l}\text { Treatment with insulin at } \\
\text { enrolment and during trial }\end{array}$ & $\begin{array}{l}\text { Digit Symbol Coding at } \\
\text { baseline and } 20 \text { and } \\
40 \text { months }\end{array}$ & $\begin{array}{l}\text { Total of } 21 \\
\text { demographic, lifestyle, } \\
\text { and clinical covariates }\end{array}$ & $\begin{array}{l}\text { Association of insulin use at } \\
\text { enrolment with lower } \\
\text { baseline cognitive function. } \\
\text { Loss of statistical significance } \\
\text { upon full adjustment. No } \\
\text { association of insulin use } \\
\text { during trial with } 40 \text {-month } \\
\text { cognitive decline in standard } \\
\text { treatment group. Association }\end{array}$ \\
\hline
\end{tabular}

duration of diabetes, Digit Symbol Coding). Greater abnormal white matter in intensively treated compared with standard treatment group 40 months. 
Table 3 Studies of hyperglycemia or hyperinsulinemia and cognitive function in type 2 diabetes (Continued)

\begin{tabular}{|c|c|c|c|c|c|c|c|c|}
\hline $\begin{array}{l}\text { Umegaki } \\
\text { et al. [16] } \\
\text { (2014) }\end{array}$ & $\begin{array}{l}\text { Patients with type } 2 \\
\text { diabetes; Japan }\end{array}$ & $\begin{array}{l}\text { 6-year } \\
\text { prospective, } \\
\text { observational }\end{array}$ & 79 & $\begin{array}{l}\text { Mean } 74 \pm \\
5 \text { years }\end{array}$ & $\begin{array}{l}\text { Mean of } \mathrm{HbA} 1 \mathrm{c} \text { and plasma } \\
\text { immunoreactive insulin at } \\
\text { baseline and annual follow-ups. }\end{array}$ & $\begin{array}{l}\text { Composite score from MMSE, } \\
\text { Digit Symbol Coding, Stroop, } \\
\text { and word recall. Analyses of } \\
\text { 'decliners' versus 'non-decliners' } \\
\text { on bases of composite score } \\
\text { and individual cognitive tests. }\end{array}$ & None & $\begin{array}{l}\text { No associations for HbA1c or } \\
\text { insulin, except for higher } \\
\text { mean 6-year insulin in } \\
\text { 'decliners' compared with } \\
\text { 'non-decliners' on Stroop. }\end{array}$ \\
\hline $\begin{array}{l}\text { Yanagawa } \\
\text { et al. [15] } \\
\text { (2011) }\end{array}$ & $\begin{array}{l}\text { Patients with } \\
\text { diabetes receiving } \\
\text { exercise program } \\
\text { four times/week } \\
\text { versus none; Japan }\end{array}$ & $\begin{array}{l}\text { 12-week trial } \\
\text { on physical } \\
\text { exercise } \\
\text { intervention }\end{array}$ & 16 & $\begin{array}{l}\text { Mean } 71 \pm \\
4 \text { years }\end{array}$ & $\begin{array}{l}\text { HbA1c, fasting blood glucose, } \\
\text { GIR, MCR in euglycemic } \\
\text { clamp, and immunoreactive } \\
\text { insulin. (No intervention effect } \\
\text { on any of these } \\
\text { measurements.) }\end{array}$ & $\begin{array}{l}\text { MMSE, word recall, Digit } \\
\text { Symbol Coding, Stroop, and } \\
\text { Trail-Making Test }\end{array}$ & $\begin{array}{l}\text { Age, education, and } \\
\text { body mass index }\end{array}$ & $\begin{array}{l}\text { No difference in cognitive } \\
\text { function between groups } \\
\text { following intervention. Across } \\
\text { groups, changes in HbA1c } \\
\text { and changes in GIR correlated } \\
\text { with changes in word recall. } \\
\text { Changes in fasting blood } \\
\text { glucose correlated with } \\
\text { changes in Trail-Making. }\end{array}$ \\
\hline
\end{tabular}

of insulin use with steeper

40-month cognitive decline

in intensive treatment group;

loss of statistical significance

upon full adjustment.

No associations for $\mathrm{HbA1c}$ or

ullin, except for higher

mean 6-year insulin in

"decliners' compared with

'decliners' versus 'non-decliners'

on bases of composite score

HbA1c, fasting blood glucose, MMSE, word recall, Digit

in euglycemic

Symbol Coding, Stroop, and

Age, education, and

No difference in cognitive

following intervention. Across

groups, changes in $\mathrm{HbA1c}$

.

changes in fasting blood

changes in Trail-Making

ACCORD-MIND, Action to Control Cardiovascular Risk in Diabetes-Memory in Diabetes; CANTAB, Cambridge Neuropsychological Test Automated Battery; CDR, Clinical Dementia Rating Scale; CIMT, carotid intima-media thickness; GIR, glucose infusion rate; $M C l$, mild cognitive impairment; $M C R$, metabolic clearance rate; $M M S E$, Mini-Mental State Examination 
Table 4 Studies of hypoglycemia and cognitive function in type 2 diabetes

Study Sample Design Number Baseline Hyp

\begin{tabular}{|c|c|c|c|c|c|c|}
\hline $\begin{array}{l}\text { Aung et al. } \\
\text { [33] (2012) }\end{array}$ & $\begin{array}{l}\text { Patients with type } 2 \\
\text { diabetes } \\
\text { participating in the } \\
\text { Edinburgh Type } 2 \\
\text { Diabetes Study; } \\
\text { Scotland }\end{array}$ & $\begin{array}{l}\text { Cross-sectional, } \\
\text { observational }\end{array}$ & 1,066 & $\begin{array}{l}\text { Mean } 68 \\
\pm 4 \text { years }\end{array}$ & $\begin{array}{l}\text { Baseline self-report of } \\
\text { history of } \mathrm{SH} \text { (defined as } \\
\text { episode requiring } \\
\text { assistance) }\end{array}$ & $\begin{array}{l}\text { MMSE, composite score } \\
\text { from seven cognitive tests, } \\
\text { and estimate of pre- } \\
\text { morbid ability }\end{array}$ \\
\hline
\end{tabular}

Bruce et al. Patients with type 2 8-year retrospective, [14] (2008) diabetes participating in the Fremantle Diabetes Study; Australia

Bruce et al. Patients with type 2 8-year retrospective, 2[21] (2008) diabetes participating in the year prospective, Fremantle Diabetes

Study; Australia

Bruce et al. Patients with type 2 5-year prospective, [34] (2009) diabetes observational participating in the Fremantle Diabetes Study; Australia
302 Mean 76 Hypoglycemia resulting in \pm 5 years coma or hospitalization, self-reported at baseline and 8 years earlier

ementia and MC identified from screening instruments/clinica interview

205 Mean 75 Hypoglycemia resulting in Dementia and $M C$ \pm 4 years coma or hospitalization, self-reported 8 years prior to baseline cognitive assessment

identified from screen

instruments/clinical

interview at baseline and at 2-year follow-up. 'Cognitive decline' defined as downward conversion between 'normal', MCl, and dementia.

302 Mean 76 1. Self-reported medical Dementia and MCI \pm 5 years assistance or unconsciousness identified from screening or both. instruments/clinica interview. 'All cognitive impairment' summarizes groups of dementia and $\mathrm{MCl}$.

2. Episodes rated by medical staff as 'doctorverified'.3. Codes for

'Cognitive decline' defined as conversion between unimpaired, $\mathrm{MCl}$, and ambulance or emergency dementia. treatment for

hypoglycemia in hospital records (HSH).
Adjustment variables

Age, sex duration of

diabetes, anti-diabetes

medication, depression hol, smoking, blood pressure, $\mathrm{HbA} 1 \mathrm{C}$, stroke, TIA Analyses of estimated lifeime decline additionally adjusted for estimated pre-morbid ability.

None

None

Association with cognitive

History of $\mathrm{SH}$ associated

with lower cognitive function and steeper estimated lifetime decline (fully adjusted analyses). Linear negative relationship between number of episodes of $\mathrm{SH}$ in the yea before cognitive testing and cognitive function (analysis controlling for age and sex).

Cross-sectional analysis: increased prevalence of history of hypoglycemia in groups with poorer cognitive function. No findings in prospective analyses.

No association

Based on preliminary associations of covariates with cognition, the following adjustment variables were selected for: analyses of MCl: age, sex education, and

cardiovascular disease; for analyses of dementia:

duration of diabetes and peripheral arterial disease; for analyses of all cognitive impairment: cardiovascular disease, peripheral arterial disease, and duration of diabetes.
Cross-sectional analyses: presence of $\mathrm{MCl}$ and all cognitive impairment associated with history of

all three measures of hypoglycemia (fully adjusted analyses). No finding for

dementia.Prospective nalyses: no association of baseline history of hypoglycemia and risk of 'cognitive decline' in patients free of cognitive impairment at baseline (unadjusted). Increased risk of first-ever incident HSH in group with dementia at baseline (adjusted for insulin use, body mass 


$\begin{array}{lll}\begin{array}{ll}\text { de Galan } \\ \text { et al. [35] }\end{array} & \text { Patients with type } 2 & \text { 5-year trial on effects of } \\ \text { (2009) } & \text { diabetes } & \text { intensified blood } \\ & \text { participating in } & \text { pressure control and } \\ & \text { ADVANCE arm on } & \text { intensified glycemic } \\ & \text { glycemic control, } & \text { control } \\ & \text { receiving standard } & \\ & \text { target versus target } \\ & \text { HbA1c } \leq 6.5 \% \text {; } & \\ & \text { Australia }\end{array}$

Feinkohl Patients with type 2 4-year prospective,

et al. [38] diabetes observational

(2014) participating in the

Edinburgh Type 2

Diabetes Study:

Scotland
11,140 Mean 66 Incident SH defined as \pm 6 years blood glucose $<2.8 \mathrm{mmo} /$ L or symptoms consisten with hypoglycemia with absence of another cause and requiring external assistance. Incident mild hypoglycemia defined as self-treated episode.

con

At baseline and 2-year intervals: MMSE followed by clinical interview for patients with MMSE <24 or suspected dementia. 'Normal' cognitive function defined as MMSE $\geq 28$; 'mild dysfunction' as MMSE = 24-27; 'severe dysfunction' as MMSE <24. continuous measure.
Age, sex, treatment arm, education, duration of diabetes, blood pressure hypertension, $\mathrm{HbA1c}$, cholesterol, body mass index, macrovascular disease, microvascular disease, smoking, and alcohol

index, inability to selfmanage medication, and history of severe

hypoglycemia). No finding on risk of subsequent hypoglycemia in patients with $\mathrm{MCl}$ at baseline.

Prospective analyses (unadjusted): increased risk of SH (but not any

hypoglycemia) in groups with 'mild dysfunction' and 'severe dysfunction' (versus 'normal' group). For 'severe dysfunction', but not 'mild dysfunction', finding survived full adjustment. Each unit-lower baseline MMSE score associated with $10 \%$ increased risk of $\mathrm{SH}$ (adjusted for age, sex, education, and treatment group). Increased risk of hypoglycemia in treatment group with intensified group with intensified
glycemic control, but finding similar across cognitive groups. No difference in cognitive decline between treatment groups.

MMSE, composite score from seven cognitive tests, and estimate of pre\pm 4 years episode requiring episode requiring (prevalent $\mathrm{SH}$ ) and during follow-up (incident SH)

Age, sex, cholesterol, blood History of SH and incident pressure, smoking, HbA1c, SH both associated with TIA, stroke, MI, and angina lower cognitive function at year 4 and with increased rate of 4-year cognitive decline. Incident SH associated with steeper estimated lifetime decline. Baseline lower cognitive function lower cognitive function incident SH. \pm 6 years hypoglycemia requiring baseline and 40 month (ACCORD- medical assistance and of MIND) episode of hypoglycemia diabetes pressure, lipids, and participating in glycemic contro

ACCORD and

with HbA1c targets

of less than $6.0 \%$

versus $7.0 \%$ to

America

Digit Symbol Coding requiring any assistance in treatment arm with

intensified glycemic (primary outcome), MMSE, Rey Auditory Verbal Learning, and Stroop (secondary outcomes) at baseline and 20 and $(n=10,251) \quad 40$ months.
Second trial assignment (lipid or blood pressure trials), group allocation

No difference in 20 - or $40-$ month cognitive decline between treatment groups in ACCORD-MIND substudy assignment clinical center, of ACCORD (fully adjusted and history of analyses). Total brain volume cardiovascular disease declined at slower rate in intensively treated compared with standard treatment group (independent of 
Punthakee Patients with type 2 40-month trial on blood et al. [36] diabetes

(2012) participating in ACCORD-MIND, with $\mathrm{HbA1c}$ targets

$7.0 \%$ to $7.9 \%$

North America pressure, lipids and

3-year retrospective

hypoglycemia Dementia

ascertained in

subsequent 4 years

glycemic control
15,000 Mean 64 ICD codes for any \pm 10 years hypoglycemia from

inpatient and outpatient medical records

2,956 Mean 62 1. SH defined as self\pm 6 years reported $<2.8 \mathrm{mmol} / \mathrm{L}$ or symptoms that resolved with use of glucose or similar.2. HMA defined as episode requiring medical assistance (hospitalization: care in emergency

department/ by emergency personnel). 3. HAA defined as episode requiring any assistance.
ICD codes for dementia Age, sex, insulin use,

from inpatient and cardiovascular disease,

outpatient medical records hypertension, ischemic heart disease, chronic kidney disease, and cholesterol

Digit Symbol Coding (Pey

Rey (secondary outcomes) at baseline and 20 and

40 months assignment (lipid or blood assignment (lipid or blood
pressure trials), group allocation within second trial assignment, duration of diabetes, stroke, $\mathrm{HbAlc}$, ethnicity, body mass index peripheral neuropathy,

urine albumin-to-creatinin ratio, and baseline use of insulin

adjustment variables, and of age, sex, duration of diabetes, and Digit Symbo Coding score). Greater abnormal white matter in intensively treated compared with standard treatment group at

$$
40 \text { months. }
$$

Hypoglycemia associated with increased risk of subsequent dementia diagnosis. Linear relationship of number of episodes with dementia risk.

Cross-sectional analyses (unadjusted): association of history of HMA with lower cognitive function at baseline. Lower baseline cognitive function predicted increased risk of first-ever HMA and HAA

(but not recurrent HMA or HAA) during follow-up across intervention groups (fully adjusted analyses). Association between 20 month cognitive decline and risk of first-ever HMA in subsequent 22 months (finding restricted to group scoring in lowest tertile of Digit Symbol Coding at baseline; analysis adjusted only for second tria assignment, group allocation within second trial assignment).

In 5- and 18-year analyses: hypoglycemia (versus none) 1980 to 2002 or

Patients with type 2 18-year retrospective,

17,000 Mean 65 ICD codes for emergency \pm 7 years treatment or hospitalization for hypoglycemia, 1980 to 1980 to 1985. ethnicity, duration of (2009)

CD codes for any outpatient medical records 2003 to 2007; additional analysis of 2005 to 2007 use, HbAlc, body mass index, with increased risk of hyperlipidemia count scores subsequent dementia of co-morbidity based on ICD (analyses of dementia 2003 codes for hypertension, car- to 2007). Association of $\geq 2$ diovascular disease stroke, episodes of hypoglycemia and end-stage renal disease (but not of single episode 


$\begin{array}{lll}\text { Yaffe et al. } & \text { Patients with } & \begin{array}{l}\text { 12-year prospective, } \\ \text { [37] (2013) }\end{array} \\ & \begin{array}{l}\text { diabetes } \\ \text { participating in } \\ \text { Health ABC; USA }\end{array} & \end{array}$

Health ABC; USA fying Partic

Participants cognitively unimpaired at baseline. Identification of dementia cases on the basis of hospital records showing ICD codes for dementia as primary or secondary diagnosis related to hospitalization, or

dementia medication on medication inventory during annual visit; MMSE administered at 2-year intervals. of hypoglycemia) versus none with increased risk dementia in anased risk of 2005 to 2007. dementia 2005 to 2007. Findings similar for codes of any hypoglycemia and for episodes resulting in hospitalization.

Cross-sectional analysis (unadjusted): association of history of hypoglycemia with lower cognitive ethnicity, diabetes baseline, insulin use, HbA1c, APOE e4 status, related to hospitalz baseline MMSE, MI, stroke,

unction Prospective analyses: hypoglycemia associated with increased risk of dementia (fully adjusted analysis; survived adjusted analysis; survived slope of MMSE over time). Dementia associated with increased risk of

hypoglycemia (analysis adjusted for adjustment variables, minus HbAr APOE e4).

ACCORD-MIND, Action to Control Cardiovascular Risk in Diabetes-Memory in Diabetes; ADVANCE, Action in Diabetes and Vascular Disease: Preterax and Diamicron Modified Release Controlled Evaluation; APOE, apolipoprotein; $H A A$, hypoglycemia needing any assistance; Health $A B C$, Health, Aging and Body Composition Study; HMA, hypoglycemia requiring medical assistance; $H S H$, health service use for hypoglycemia; $I C D$, International Classification of Diseases; MCI, mild cognitive impairment; $M I$, myocardial infarction; MMSE, Mini-Mental State Examination; SH, severe hypoglycemia; TIA, transient ischemic attack 
dyslipidemia as a potential risk factor for diabetes-related cognitive impairment (Table 2). Cross-sectional studies have revealed trends for increased prevalence of hypertension in patients with lower cognitive function $[11,13,19$, 20], but cross-sectional analyses of blood pressure as a continuous measure have failed to identify similar associations $[11,14,21]$. On the other hand, some $[14,19,22]$, though not all $[17,21]$, prospective studies have found a relationship between baseline blood pressure or hypertension and the subsequent risk of cognitive decline. In the Fremantle Diabetes Study, higher baseline diastolic blood pressure was associated with an increased risk of incident $\mathrm{AD}$ after 8 years [14], and in an investigation of people over 80 years of age, the coexistence of hypertension appeared to exacerbate diabetes-related cognitive decline during a 6-year follow-up and to increase the risk of dementia [19]. Similarly, a retrospective study that examined the hospital records of almost 380,000 older patients with diabetes showed that co-morbid hypertension increased the 2-year risk of dementia; treatment with anti-hypertensive medication (other than $\alpha$-adrenoceptor blockers, with which the risk of dementia was increased) further diminished the risk of dementia by between $4 \%$ and $24 \%$ depending on the precise type of drug used [22]. In contrast with these findings, the blood pressure trial of the ACCORD-MIND study did not demonstrate a difference in cognitive decline over a period of 40 months between a group of patients who received intensive anti-hypertensive therapy and a group on conventional treatment, despite the success of the trial in producing a difference in blood pressure between the two treatment groups [17]. However, a direct association between blood pressure and cognitive decline was not explored. In the general (non-diabetic) population, the results of observational studies and of RCTs investigating links between hypertension and cognitive impairment have also, in the main, been negative $[2,23]$. Therefore, although hypertension causes cerebrovascular disease and, as such, represents a good candidate for a cognitive risk factor, its role in the development of cognitive decline during aging in either the diabetic or non-diabetic population remains unproven.

\section{Hyperglycemia}

Raised blood glucose levels within the non-diabetic or pre-diabetic range have consistently been associated with cognitive impairment, with the strength of the association increasing with advancing age [24]. Given that diabetes is characterized by persistently raised blood glucose levels, a causative role for hyperglycemia in diabetes-associated cognitive decline would seem likely. However, the findings from cross-sectional analyses on the association of HbA1c with cognitive function $[9,11,13,14,20,25]$ and cognitive decline $[14,16,21]$ in people with type 2 diabetes have been inconsistent (Table 3), potentially due to the different ages of the study populations. Overall, the association of type 2 diabetes with increased cognitive impairment appears to be relatively weak before the age of 70 years, provided that good glycemic control is maintained, and it is only in older patients that cognitive decrements related to chronic hyperglycemia become apparent [26]. More recently, a retrospective analysis of a cohort of people with type 2 diabetes, in whom 12-year data on HbA1c were available from a diabetes register, showed that in addition to increments in blood glucose levels over time, poor glycemic control long-term predicted a lower level of late-life cognitive function, despite a trend toward improved glycemic control by intensifying therapy [27]. This is consistent with the evidence showing damaging effects of mid-life diabetes on the risk of late-life cognitive impairment $[4,5]$ and suggests that irreversible damage may already have occurred to predispose people to cognitive impairment by the time that aggressive glucoselowering treatment was commenced.

In one of a number of intervention studies, changes in blood glucose levels due to physical exercise correlated with changes in cognitive function [15]. The ACCORDMIND study also found a statistically non-significant trend for decelerated decline in processing speed at 20 months in the group with intensive therapy for glycemic control (who achieved relatively greater glycemic control) as compared with the conventional treatment group (with resulting poorer glycemic control), although this difference was no longer apparent at 40 months [28]. Two smaller trials of patients with type 2 diabetes have reported significant associations between improved glycemic control and cognitive function. In one, improvements in glycemic control in both treatment groups due to treatment with either rosiglitazone or glibenclamide (glyburide) correlated with improvement in working memory over a period of 24 weeks [29]. In another, a reduction in post-prandial glucose excursions with repaglinide was associated with a decline in cognitive function over a period of 12 months compared with subjects who received glibenclamide and did not show such a change in glucose excursions; the decline in HbA1c was of similar magnitude in the two treatment groups, suggesting a specific role for post-prandial glucose excursions [30]. Whereas overall a recent systematic review combining the evidence from observational studies and from RCTs concluded that both hyperglycemia and glucose excursions are weakly associated with poorer cognitive function in people with type 2 diabetes [31], a meta-analysis restricted to RCTs suggested that improvement in glycemic control was unrelated to cognitive decline [32], illustrating the need for further evaluation of hyperglycemia as a potentially modifiable cognitive risk factor. 


\section{Hypoglycemia}

Few studies have investigated the effect of previous exposure to recurrent hypoglycemia on cognitive function in people with type 2 diabetes. Heterogeneity with respect to how 'hypoglycemia' has been defined presents a major problem for interpretation of results, with recorded events ranging from asymptomatic biochemical hypoglycemia to severe disabling hypoglycemia (Table 4).

Cross-sectional analyses have reported an association between a history of previous self-reported or medically verified severe hypoglycemia, defined as any episode requiring external help to effect recovery, and cognitive impairment $[14,33,34]$ but this could reflect lower cognitive ability in people who go on to experience a higher frequency of severe hypoglycemia. Indeed, in the Action in Diabetes and Vascular Disease: Preterax and Diamicron Modified Release Controlled Evaluation (ADVANCE) trial, which successfully manipulated the level of glycemic control (intensive versus standard) in patients with type 2 diabetes, each one-unit-lower score on a cognitive screening instrument at baseline was associated with a $10 \%$ greater risk of severe hypoglycemia during follow-up [35]. A lower baseline cognitive function and (for participants who had low processing speed at baseline) a relatively steeper cognitive decline between baseline and the 20-month assessment were also predictive of an increase in the subsequent first-ever hospital admission to treat severe hypoglycemia in ACCORD-MIND, and the group with low processing speed and declining cognitive function had a higher cumulative incidence of severe hypoglycemia over the 4 years of the study [36]. Finally, in two further prospective investigations, a baseline diagnosis of dementia or a diagnosis during the follow-up period in previously unimpaired participants was associated with a two- to three-fold higher rate of hospital admission for emergency medical treatment of hypoglycemia during follow-up [34, 37].

Whether exposure to hypoglycemia precedes cognitive decline and may even be a causal risk factor for this condition is less clear. In the Edinburgh Type 2 Diabetes Study (ET2DS) of more than 1,000 adults between 60 and 75 years of age, a history of severe hypoglycemia was associated with lower cognitive function when the estimated pre-morbid cognitive function before exposure to hypoglycemia was compared with post-hypoglycemia cognitive function, with evidence of an acceleration of late-life cognitive decline that was independent of the potential influence of further episodes of hypoglycemia [38] (Fig. 2). However, these observations conflict with the findings of the Fremantle Diabetes Study [21] and with the evidence from RCTs. In ACCORD-MIND and ADVANCE, cognitive function declined at similar rates by 40 months and 5 years in patients in the intensive treatment groups (in whom the incidences of hypoglycemia

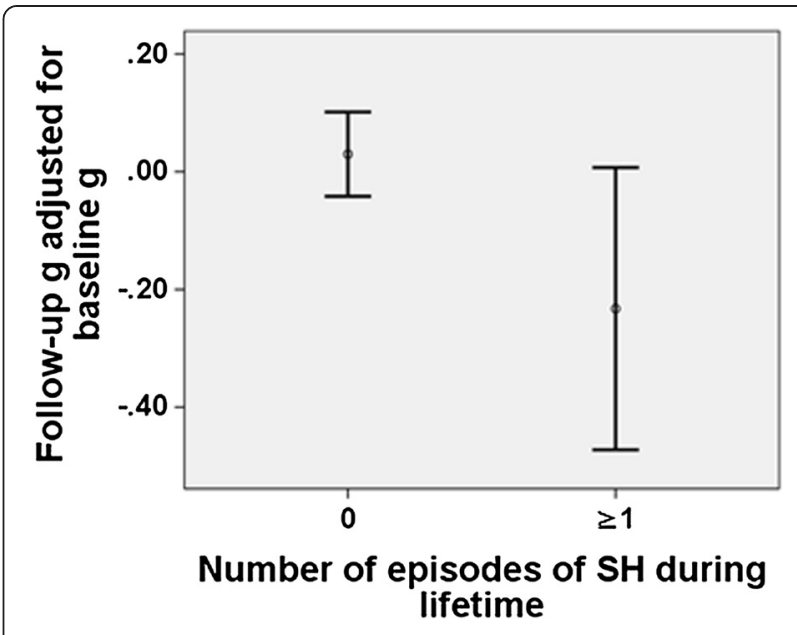

Fig. 2 Relationship of severe hypoglycemia with cognitive decline in the Edinburgh Type 2 Diabetes Study. Relationship of a self-reported history of one or more episodes of severe hypoglycemia $(\mathrm{SH})$ (defined as requiring external help) prior to the baseline clinic of the Edinburgh Type 2 Diabetes Study with the subsequent decline on a composite measure (' $g$ ') of cognitive ability during 4-year follow-up. Error bars show $95 \%$ confidence interval $(n=831)$. Based on [38]

were significantly higher) compared with those in the standard treatment arms $[28,35]$. However, in both of these trials, the management of diabetes was manipulated to attain pre-determined glycemic targets. It is plausible that any detrimental effect of hypoglycemia was counterbalanced by an improvement in cognitive function occurring through specific beneficial effects of the assigned intervention.

Two retrospective investigations $[39,40]$ have suggested that a dose-response relationship may exist between the frequency of exposure to severe hypoglycemia and the subsequent risk of dementia. However, these studies relied on hospital records, a suboptimal method of identifying hypoglycemia, and the suggestion that exposure to a single episode of hypoglycemia would induce dementia is biologically implausible. In the observational analysis of the Fremantle Diabetes Study, a history of severe hypoglycemia failed to predict the 5-year risk of dementia [34]. In the prospective Health Aging and Body Composition Study, participants with incident hypoglycemia had a two-fold risk of subsequent dementia over a period of 12 years, but in combination with the analysis showing an increased risk of subsequent hypoglycemia in patients who were diagnosed with dementia during follow-up, the data were overall interpreted as showing bidirectional causality [37]. It is essential that the role of hypoglycemia in either causing or accelerating cognitive decline be clarified in view of the current policy to use intensive therapy to achieve near-normoglycemia to minimize the development of diabetic complications. 


\section{Hyperinsulinemia}

Hyperinsulinemia from endogenous hypersecretion of insulin is common in the early stages of type 2 diabetes as a 'pathophysiological' response to insulin resistance; it also occurs as a consequence of exogenous insulin therapy. Hyperinsulinemia has been associated with cognitive impairment, but a systematic review of observational studies that included people with and those without diabetes concluded that the evidence for an association of elevated plasma insulin concentrations with impairment of cognition was weak, because it is possible that any association of plasma insulin with cognition in such samples had been influenced by the inclusion of people with diabetes [24]. Very few studies have been performed in non-diabetic populations or exclusively in people with type 2 diabetes (Table 3). In one observational study, a higher mean insulin during a 6-year period was associated with a steeper rate of concurrent cognitive decline based on a test of executive function [16], and in a small intervention study, which was unsuccessful in inducing a difference in insulin sensitivity in two treatment groups through physical exercise, improvement in memory performance correlated with improvements in insulin resistance [15]. By contrast, in a larger 24-week trial in middle-aged to older patients with type 2 diabetes (mean age of 60 years), an improvement in plasma insulin levels and insulin sensitivity had no effect on concurrent change in cognitive function [29]. Similarly, in the ACCORD-MIND study, treatment with insulin on study entry or during the trial was relatively unrelated to 40-month cognitive change, but plasma insulin levels as such were not considered [41]. This is despite the fact that compliance by participants is difficult to ascertain. Inter-relationships between plasma insulin concentration, insulin resistance, and the quality of glycemic control further complicate attempts to evaluate associations of any of these risk factors with cognitive impairment.

\section{Inflammation}

Chronic low-grade inflammation is a characteristic feature of both diabetes and $\mathrm{AD}$ and appears to interact with diabetes in its association with cognitive impairment. This suggests a common biological mechanism [42]. Circulating markers of inflammation include C-reactive protein (CRP), interleukin-6 (IL-6), fibrinogen, and tumor necrosis factor-alpha (TNF- $\alpha$ ), some of which have been associated with cognitive dysfunction in people with diabetes (Additional file 1: Table S1). Elevated levels of CRP have been associated with lower cognitive function in small studies of hospitalized patients (for example, [9]). In the ET2DS, higher levels of fibrinogen, TNF- $\alpha$, and IL-6 but not CRP were associated with lower measures of cognitive function [43, 44]; higher baseline levels of fibrinogen and IL-6 additionally predicted a steeper 4-year cognitive decline $[45,46]$. CRP levels were also unrelated to cognitive decline in a further prospective study with a 6-year follow-up [16]. In support of associations (particularly, causal) between inflammation and cognition, genetic variants that influence circulating levels of inflammatory markers have been associated with cognitive impairment, but this finding has not been consistent $[43,47]$.

\section{Microvascular disease}

Because of the homology between retinal and cerebrovascular cells, the state of small vessels in the retina closely mirrors that of the cerebral microvasculature, suggesting that diabetic retinopathy can be used as a marker for the presence of microangiopathy within the brain. A systematic review of cross-sectional and prospective observational studies concluded that people from the general population and people with diabetes who exhibit retinal microvascular abnormalities appear to be at increased risk of cognitive impairment, including dementia, compared with people who have no retinal microvascular abnormalities [48], although subsequent studies have given conflicting results [25, 35, 49] (Additional file 1: Table S2). However, in support of the findings of the systematic review, baseline presence of retinopathy was recently identified as a predictor of steeper rates of cognitive decline during 40-month (but not 20-month intermittent) follow-up in ACCORD-MIND [50]. Overall, diabetic retinopathy may be a putative surrogate marker for cognitive impairment in people with diabetes, in which cerebral microvascular disease may have an important pathogenetic role.

\section{Macrovascular disease}

The prevalence of both symptomatic and asymptomatic macrovascular disease is increased in people with type 2 diabetes. Given the likely links between vascular and cognitive pathologies, markers of such vascular 'endorgan damage' have the potential to identify a group of subjects who are at particularly high risk of developing cognitive impairment. Assessing the association between different macrovascular diseases and cognitive impairment may also help us understand underlying pathophysiological mechanisms. To this end, it is interesting to consider the extent to which studies have uncovered associations of cognitive impairment with specific types of macrovascular disease, such as coronary heart disease (CHD), cerebrovascular disease, and peripheral arterial disease, and with vascular biomarkers which indicate underlying subclinical macrovascular disease in the related vascular trees.

\section{Coronary heart disease and $\mathrm{N}$-terminal pro-brain natriuretic peptide}

An association of CHD with a lower level of cognitive function was observed in the ET2DS [51] (Additional file 
1: Table S3). However, in the ACCORD-MIND study, the evidence for an association of CHD with cognitive dysfunction was limited [13], and in all other crosssectional investigations $[14,35]$ and in all prospective analyses, including the ET2DS [21, 51], the results have been negative. The only significant prospective association was in the direction of cognitive ability predicting worsening of CHD. In ADVANCE, after multivariate adjustment, a baseline presence of 'mild cognitive dysfunction' and 'severe cognitive dysfunction' increased the 5 -year risk of a major coronary event by $31 \%$ and $70 \%$, respectively [35].

The inactive metabolite $\mathrm{N}$-terminal pro-brain natriuretic peptide (NT-proBNP) is a biomarker of the cardiac stress associated with ventricular dysfunction and congestive heart failure. In the ET2DS, an association of small effect size was found between a higher baseline NT-proBNP and a lower cognitive ability and with a steeper cognitive decline later in life [51] (Additional file 1: Table S4). In the general population and in people with cardiovascular disease, associations of small to large unadjusted effect size have been reported relatively consistently between elevated levels of natriuretic peptide and lower cognitive function (for example, [52]) and with the presence of dementia or milder forms of impairment $[53,54]$. Null findings are rare [55] and in some studies may have resulted from the nature of the cognitive screening instruments that were applied. Some results have suggested an independence of these associations from symptomatic macrovascular disease, including stroke [52], which was also observed in the ET2DS [51]. Prospective investigations of the general population were, until recently, restricted to a single cohort (of people over 75 years of age) in which the findings were inconclusive $[53,54]$. However, a recent large Finnish study ( $n=7,000$ participants) that examined the relationship of natriuretic peptides with cognitive function has not provided definitive evidence [56]. In that study, each standard deviation above baseline NT-proBNP predicted a $48 \%$ increased risk of dementia during 14-year follow-up after multivariate adjustment in men, but no such association was found in women.

\section{Cerebrovascular disease and carotid intima-media thickness}

An association between a lower level of cognitive function and cerebral infarction has been a consistent finding in populations with diabetes $[13,51]$ (Additional file 1: Table S3). For example, in the Fremantle Diabetes Study, cognitive impairment diagnosed on the basis of a screening instrument and follow-up clinical interview was associated with a history of cerebrovascular disease [14]. The impact of stroke on cognitive function was demonstrated in the diabetic subpopulation of a Dutch study and in the ET2DS, in which an association between stroke and diminished cognitive function persisted after adjustment for estimated pre-morbid ability $[20,25,51]$.

In the ET2DS and in the Fremantle Diabetes Study, a history of stroke was associated with a steeper decline in cognitive ability $[14,51]$, but this observation differed from those of several other prospective analyses (for example, [21]), in which no such association was found. In the ADVANCE study, evidence of a prospective association in the direction of lower cognitive function predisposing patients to an increased risk of infarction was observed. After multivariate adjustment, individuals with 'mildly reduced' cognitive function at baseline had a 5 -year risk of sustaining a major stroke which was $34 \%$ greater than that of individuals who had a higher level of cognitive ability; people with 'severe cognitive dysfunction' had a $71 \%$ greater risk [35]. The relationship between cerebral infarction and cognition in diabetes may therefore be bidirectional.

In people with type 2 diabetes, greater carotid intimamedia thickness (cIMT) has been associated with a lower level of cognitive function $[9,11]$, but its association with an estimated steeper decline of lifetime cognitive function has been inconsistent $[25,51]$ (Additional file 1: Table S3). To date, the ET2DS appears to be the only prospective study to examine cIMT and cognition in people with type 2 diabetes. This identified an association of cIMT with a steeper decline in late-life cognitive function, which was independent of a preceding history of stroke [51]. In the population in general, an association between a higher cIMT and an increased risk of cognitive impairment has been established [57], and so a similar association is likely to exist in people with type 2 diabetes.

\section{Peripheral arterial disease and ankle-brachial pressure index}

In people with type 2 diabetes, a low ankle-brachial pressure index (ABI) - a measure of peripheral arterial disease (PAD) of the lower limbs and of more generalized atherosclerosis - and PAD diagnosis have been associated with lower cognitive function $[9,51]$ and with dementia [14] (Additional file 1: Table S3). In the Fremantle Diabetes Study, $38 \%$ of cognitively 'normal' individuals, $45 \%$ of people with reduced cognitive function, and $75 \%$ of people with frank dementia had evidence of coexisting PAD [14]. In one study, cross-sectional findings for 'any vascular event' (which was partly defined by PAD) remained significant after adjustment for an estimate of peak pre-morbid ability [20, 25], but after such an adjustment was made in the analysis of ABI and symptomatic PAD in the ET2DS, it did not quite achieve statistical significance [51]. However, in the latter, each standard deviation of a lower baseline ABI was also associated with a 0.12-standard deviation increment in subsequent 4-year decline on a composite measure of cognitive function [51]. In the Fremantle 
Diabetes Study, PAD measured 8 years earlier also predicted an increased risk of cognitive impairment [14] though it was not associated with the risk of cognitive decline in the subsequent 2-year follow-up period [21].

Overall, the evidence for an association between macrovascular disease and cognitive impairment in diabetes is inconsistent and varies according to the area of the vasculature considered. As might be expected, evidence for a relationship with cerebrovascular disease, especially stroke, is stronger than that for vascular sites which are more distant from the brain, including the heart. Evidence for an association with the most distal presentations of macrovascular disease, such as PAD of the lower limbs, is particularly limited, is likely to reflect widespread atherosclerosis as a marker for cognitive impairment in people with diabetes, and would suggest that any true associations have a small effect size.

\section{Depression and pre-morbid cognitive ability Depression}

Cross-sectional studies of cognitive function in people with diabetes, with or without depressive symptoms or clinical depression, have been inconclusive (Additional file 1: Table S5). One investigation of older people with type 2 diabetes reported a statistically non-significant trend for negative correlations between scores on a cognitive screening instrument and scores on a self-administered screening instrument for depression [58]. In a crosssectional analysis of ACCORD-MIND, patients with depression (based on scores on screening instrument or on self-report) also scored lower on a cognitive screening instrument (though not on more detailed neuropsychological tests) compared with patients who were free of depression [13]. Additive detrimental effects have been suggested by another study of people with type 2 diabetes and healthy controls who were 30 to 80 years of age (the mean age was 60 years across groups), to whom more detailed neuropsychological testing was applied along with clinical interviews to diagnose depression. The patients with co-morbid diabetes and depression performed less well on tests of attention and processing speed compared with participants with diabetes but without depression. Relative to the latter, there was also a trend just short of statistical significance for lower cognitive function overall in the group with co-morbid diabetes and depression [59]. In a prospective analysis of a large cohort of Americans, co-morbidities of diabetes and depression were also linked to a $100 \%$ increased risk of dementia over a period of 3 to 5 years when compared with people with diabetes but without depression [60]. Finally, ACCORD-MIND revealed associations of higher scores on a screening instrument for depression and a steeper 40-month cognitive decline [61]. In the general population, the association of depression with cognitive impairment appears to be well established [60], and so it seems likely that depression has a contributing role in promoting diabetes-associated cognitive impairment.

\section{Pre-morbid cognitive ability}

Diabetes-associated cognitive impairment may partly reflect reverse causality. Consistent with the assumption that individuals who have lower cognitive ability may be predisposed to have lower late-life cognitive function and to be at increased risk of developing diabetes as they get older, an analysis of the Lothian Birth Cohort (a group of people who were born in 1936) found that cross-sectional associations of diabetes with lower latelife cognitive ability disappeared following adjustment for cognitive ability that had been measured at age 11 [62] (Fig. 3).

However, where diabetes is associated with a steeper late-life cognitive decline in prospective analyses, the role of pre-morbid ability is as yet unclear, particularly as its role in promoting late-life cognitive decline per se is uncertain. Some prospective investigations have indicated that individuals with lower cognitive ability decline more rapidly as they get older [63], but this has not been confirmed [64].

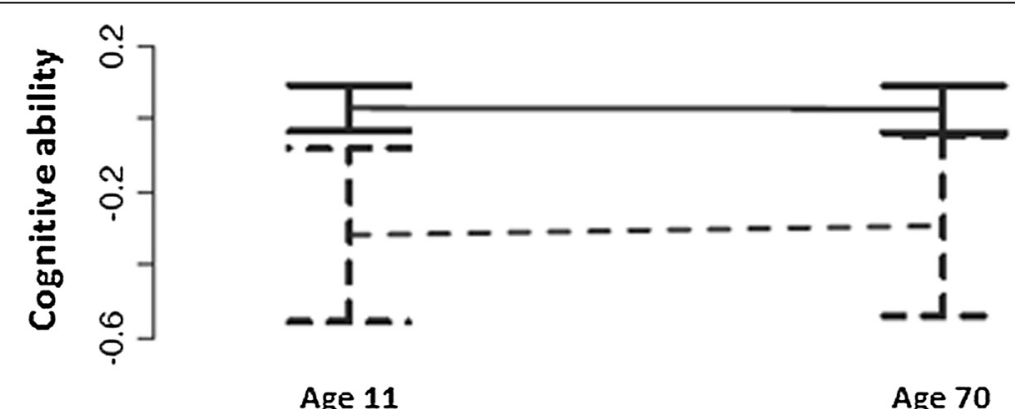

Fig. 3 Mean difference (95\% confidence interval) in cognitive test scores in people with and without diabetes at ages 11 and 70 in the Lothian Birth Cohort (1936). Solid lines reflect people without diabetes at age 70; dashed lines reflect people with diabetes at age 70 . Reproduced with permission from Elsevier [62] 


\section{Neuropathological effects}

The neuropathological features of $\mathrm{VaD}$ (multiple infarcts) and $\mathrm{AD}$ (cerebral plaques of beta amyloid and hyperphosphorylated tau contributing to neurofibrillary tangles) [65] are well established. Increasingly, it is being recognized that there may be considerable overlap in the etiology of these two conditions [66, 67], and individuals with cognitive decline often exhibit both pathologies. Many of the risk factors reviewed in this article have the potential to contribute to such neuropathology. Clearly, it is not difficult to conceive how the macrovascular risk factors in diabetes would contribute to cerebrovascular damage [12], while chronic hyperglycemia may lead to the accumulation of advanced glycation end-products in the brain [68] and the development of small vessel ischemic change. The neurotoxic effects of hypoglycemia are also well understood [8], and there are strong links between insulin and beta amyloid: insulin appears to initiate the production of beta amyloid as well as promote its accumulation through competition for degradation by insulin-degrading enzyme [67]; it may further contribute to amyloid formation through co-secretion of the amyloidforming peptide amylin with insulin from pancreatic beta cells $[65,69]$. Consistent with amylin being a neuropathological mediator of associations between diabetes and cognitive impairment, a recent post-mortem study demonstrated the presence of the peptide in the brains of people with diabetes and in those with AD, but not in healthy controls [69]. Additional associations of amylin with vascular damage [69] are consistent with the premise that $\mathrm{AD}$ and $\mathrm{VaD}$ may not be as clearly distinct as has been thought previously.

As becomes clear, the neuropathological bases of the increased risk of cognitive impairment that people with diabetes are exposed to are far from singular and straightforward. Rather, highly complex, cell-level processes appear to be at play. It is this complexity which explains the difficulty in the development of effective strategies for prevention of cognitive impairment in people with diabetes and in the development of treatment approaches in those patients who have already become cognitively impaired.

\section{Conclusions}

Most studies that have addressed the risk factors associated with cognitive impairment have examined cohorts from the general population. However, in view of the greater risk of cognitive impairment affecting people with type 2 diabetes and the potential differences in underlying mechanisms between people with type 2 diabetes and the general population, more information that is specific to diabetic populations is required, particularly in older adults. The evidence that risk factors that occur more frequently in people with type 2 diabetes are associated with cognitive impairment is limited, mainly because few of these risk factors have been investigated in any depth. Many have also been assessed in isolation. The evidence that is currently available points to a role for poor glycemic control, hypoglycemia, micro- and macrovascular disease, inflammation, and depression as potential risk factors for cognitive impairment in people with diabetes. However, the causality in these relationships is less clear. The roles of dyslipidemia, hyperinsulinemia, hypertension, and pre-morbid ability as putative risk factors are as yet undetermined and require further investigation. Overall, we would recommend that clinicians temper the current emphasis on intensive therapy and strict glycemic control in an attempt to protect the cognitive function of their patients (particularly in view of the potentially detrimental effects that hypoglycemia may have on cognition). We would encourage them to take a holistic approach to patient management by addressing the full range of modifiable risk factors while being aware of the potential influences of risk factors for cognitive impairment that are not modifiable.

A previous review of research in this field [70] has indicated that evidence has advanced mainly in a quantitative manner in recent decades. For modifiable risk factors, further high-quality and large-scale trials are needed to determine causality in the interaction between each major risk factor and their association with cognitive decline. For glycemic control, future trials should continue to attempt to separate out the potential duality of beneficial (reduced blood glucose levels) and detrimental (hypoglycemia) effects. Rather than using statistical adjustment methods, such as controlling for hypoglycemia in analyses of anti-diabetes agents and cognitive decline, effects of anti-diabetes agents that do not induce hypoglycemia could be investigated for that purpose.

Novel directions could also be taken to investigate risk factors for which the evidence has been largely restricted to observational studies despite being modifiable. For instance, trials could determine effects of anti-inflammatory medication such as non-steroidal anti-inflammatory drugs, which are already relatively widely used and are low in cost, in order to provide definitive evidence on potential associations of these risk factors with cognitive impairment in people with diabetes as have become apparent from some observational investigations.

Undoubtedly, large trials are difficult and costly to conduct, not least because they are resource-intensive, and for non-modifiable risk factors are not always possible. As a consequence, cohort studies are likely to continue dominating this field of research. Harmonization of risk factor assessments and methodologies between cohorts should be sought with the aim of enabling integration of a range of cohorts into single large-scale analyses. Instead of focusing on individual risk factors with resultant data 'slicing', 
investigators should ascertain the inter-relationships among a range of risk factors and should explore their temporal developments. Specifically, future cohort studies, including birth cohorts, could use multi-wave designs to allow statistical procedures such as latent growth curve modeling to determine the probable inter-relationships among putative risk factors and establish their true associations (if any) with cognitive decline. In view of recent evidence of an association between cognitive impairment and brain atrophy in mid-life diabetes [71], the age at which individuals are recruited for cohort studies may have to be reconsidered to enable a life-course approach to this issue. It is to be hoped that ongoing and future research will identify causal risk factors that can be used to develop preventative interventions and help to identify which patients are at greatest risk of developing cognitive impairment.

Note: This article is part of a series on The impact of acute and chronic medical disorders on accelerated cognitive decline, edited by Carol Brayne and Daniel Davis. Other articles in this series can be found at http://alres.com/series/medicaldisorders.

\section{Additional file}

Additional file 1: Tables S1-S5. A summary of the evidence reviewed in this article on associations of inflammation, microvascular disease, macrovascular disease, natriuretic peptides and depression with cognitive function.

\section{Abbreviations \\ ABI: ankle-brachial pressure index; ACCORD-MIND: Action to Control Cardiovascular Risk in Diabetes-Memory in Diabetes; AD: Alzheimer's disease; ADVANCE: Action in Diabetes and Vascular Disease: Preterax and Diamicron Modified Release Controlled Evaluation; CHD: coronary heart disease; CIMT: carotid intima-media thickness; CRP: C-reactive protein; ET2DS: Edinburgh Type 2 Diabetes Study; IL-6: interleukin-6; NT-proBNP: $\mathrm{N}$-terminal pro-brain natriuretic peptide; PAD: peripheral arterial disease; $\mathrm{RCT}$ : randomized controlled trial; TNF-a: tumor necrosis factor alpha; VaD: vascular dementia.}

\section{Competing interests}

MWJS has received speaking honoraria from Novo Nordisk (Bagsværd, Denmark), Eli Lilly and Company (Indianapolis, IN, USA), Pfizer (New York, NY, USA), and Bristol-Myers Squibb (New York, NY, USA) and has received support from Sanofi (Paris, France) to attend a scientific meeting. He has also participated in a GlaxoSmithKline (Brentford, UK) data monitoring committee. BMF has received honoraria as a speaker at scientific meetings organized by Novo Nordisk, Eli Lilly and Company, Sanofi, MSD (Kenilworth, NJ, USA), Boehringer Ingelheim (Ingelheim, Germany), and Janssen (Beerse, Belgium) and has served on advisory boards for Novo Nordisk, Eli Lilly and Company, Sanofi, MSD, Boehringer Ingelheim, and Janssen. JFP and IF declare that they have no competing interests.

\section{Author details}

${ }^{1}$ Centre for Population Health Sciences, Medical School, Teviot Place, Edinburgh EH8 9AG, Scotland, UK. ${ }^{2}$ Metabolic Unit, Western General Hospital, Crewe Road South, Edinburgh EH4 2XU, Scotland, UK. ${ }^{3}$ The Queen's Medical Research Institute, University of Edinburgh, College of Medical and Veterinary Medicine, 47 Little France Crescent, Edinburgh EH16 4TJ, Scotland, UK.
Published online: 10 June 2015

\section{References}

1. Lu F-P, Lin K-P, Kuo H-K. Diabetes and the risk of multi-system aging phenotypes: a systematic review and meta-analysis. PLoS One. 2009;4, e4144.

2. Cooper C, Sommerlad A, Lyketsos CG, Livingston G. Modifiable predictors of dementia in mild cognitive impairment: a systematic review and meta-analysis. Am J Psychiatry. 2015;172:323-34.

3. Cukierman T, Gerstein HC, Williamson JD. Cognitive decline and dementia in diabetes - systematic overview of prospective observational studies. Diabetologia. 2005;48:2460-9.

4. Winkler A, Dlugaj M, Weimar C, Jöckel K-H, Erbel R, Dragano N, et al. Association of diabetes mellitus and mild cognitive impairment in middle-aged men and women. J Alzheimers Dis. 2014:42:1269-77.

5. Rawlings AM, Sharrett AR, Schneider AL, Coresh J, Albert M, Couper D, et al. Diabetes in midlife and cognitive change over 20 years: a cohort study. Ann Intern Med. 2014;161:785-93.

6. Sato N, Morishita R. Roles of vascular and metabolic components in cognitive dysfunction of Alzheimer disease: short- and long-term modification by non-genetic risk factors. Front Aging Neurosci. 2013;5:1-9.

7. Glass CK, Saijo K, Winner B, Marchetto MC, Gage H. Mechanisms underlying inflammation in neurodegeneration. Cell. 2010;140:918-34.

8. Arbelaez AM, Hershey T. Imaging hypoglycemia's effects on the human brain. Diabet Hypoglycemia. 2010;3:3-9.

9. Chen G, Cai L, Chen B, Liang J, Lin F, Li L, et al. Serum level of endogenous secretory receptor for advanced glycation end products and other factors in type 2 diabetic patients with mild cognitive impairment. Diabetes Care. 2011:34:2586-90.

10. Perlmutter LC, Nathan DM, Goldfinger SH, Russo PA, Yates J, Larkin M. Triglyceride levels affect cognitive function in noninsulin-dependent diabetics. J Diabet Complications. 1988:2:210-3.

11. Chen RH, Jiang XZ, Zhao XH, Qin YL, Gu Z, Gu PL, et al. Risk factors of mild cognitive impairment in middle aged patients with type 2 diabetes: a cross-section study. Ann Endocrinol. 2012;73:208-12.

12. Van Harten B, Oosterman J, Muslimovic D. Potter van Loon B-J, Scheltens P, Weinstein HC. Cognitive impairment and MRI correlates in the elderly patients with type 2 diabetes mellitus. Age Ageing. 2007;36:164-70.

13. Cukierman-Yaffe T, Gerstein HC, Williamson JD, Lazar RM, Lovato L, Miller $M E$, et al. Relationship between baseline glycemic control and cognitive function in individuals with type 2 diabetes and other cardiovascular risk factors: the action to control cardiovascular risk in diabetes-memory in diabetes (ACCORD-MIND) trial. Diabetes Care. 2009;32:221-6.

14. Bruce DG, Davis WA, Casey GP, Starkstein SE, Clarnette RM, Foster JK, et al. Predictors of cognitive impairment and dementia in older people with diabetes. Diabetologia. 2008;51:241-8.

15. Yanagawa M, Umegaki H, Uno T, Oyun K, Kawano N, Maeno H, et al. Association between improvements in insulin resistance and changes in cognitive function in elderly diabetic patients with normal cognitive function. Geriatr Gerontol Int. 2011;11:341-7.

16. Umegaki H, Kawamura T, Umemura T, Kawano N. Factors associated with cognitive decline in older adults with type 2 diabetes mellitus during a 6-year observation. Geriatr Gerontol Int. 2014;15:302-10.

17. Williamson JD, Launer LJ, Bryan RN, Coker LH, Lazar RM, Gerstein HC, et al. Cognitive function and brain structure in persons with type 2 diabetes mellitus after intensive lowering of blood pressure and lipid levels: a randomized clinical trial. JAMA Intern Med. 2014;174:324-33.

18. Van Vliet P. Cholesterol and late-life cognitive decline. J Alzheimers Dis. 2012;30:S147-62.

19. Hassing LB, Hofer SM, Nilsson SE, Berg S, Pedersen NL, McClearn G, et al. Comorbid type 2 diabetes mellitus and hypertension exacerbates cognitive decline: evidence from a longitudinal study. Age Ageing. 2004;33:355-61.

20. Manschot SM, Brands AM, van der Grond J, Kessels RP, Algra A, Kappelle LJ, et al. Brain magnetic resonance imaging correlates of impaired cognition in patients with type 2 diabetes. Diabetes. 2006;55:1106-13.

21. Bruce DG, Davis WA, Casezy GP, Starkstein SE, Clarnette RM, Almeida OP, et al. Predictors of cognitive decline in older individuals with diabetes. Diabetes Care. 2008;31:2103-7.

22. Johnson ML, Parikh N, Kunik ME, Schulz PE, Patel JG, Chen H, et al. Antihypertensive drug use and the risk of dementia in patients with diabetes mellitus. Alzheimer's Dement. 2012;8:437-44. 
23. Qiu C, Winblad B, Fratiglioni L. The age-dependent relation of blood pressure to cognitive function and dementia. Lancet Neurol. 2005;4:487-99.

24. Lamport DJ, Lawton CL, Mansfield MW, Dye L. Impairments in glucose tolerance can have a negative impact on cognitive function: a systematic research review. Neurosci Biobehav Rev. 2009;33:394-413.

25. Manschot SM, Biessels GJ, de Valk H, Algra A, Rutten GE, van der Grond J, et al. Metabolic and vascular determinants of impaired cognitive performance and abnormalities on brain magnetic resonance imaging in patients with type 2 diabetes. Diabetologia. 2007;50:2388-97.

26. Messier C. Impact of impaired glucose tolerance and type 2 diabetes on cognitive aging. Neurobiol Aging. 2005;26:26-30.

27. Ravona-Springer R, Heymann A, Schmeidler J, Moshier E, Godbold J, Sano $\mathrm{M}$, et al. Trajectories in glycemic control over time are associated with cognitive performance in elderly subjects with type 2 diabetes. PLoS One. 2014;9, e97384.

28. Launer LJ, Miller ME, Williamson JD, Lazar RM, Gerstein HC, Murray AM, et al. Effects of intensive glucose lowering on brain structure and function in people with type 2 diabetes (ACCORD MIND): a randomised open-label substudy. Lancet Neurol. 2011;10:969-77.

29. Ryan CM, Freed MI, Rood JA, Cobitz AR, Waterhouse BR, Strachan MW. Improving metabolic control leads to better working memory in adults with type 2 diabetes. Diabetes Care. 2006;29:345-51.

30. Abbatecola AM, Rizzo MR, Barbieri M, Grella R, Arciello A, Laieta MT, et al. Postprandial plasma glucose excursions and cognitive functioning in aged type 2 diabetics. Neurology. 2006;67:235-40.

31. Geijselaers SL, Sep SJ, Stehouwer CD, Biessels GJ. Glucose regulation, cognition, and brain MRI in type 2 diabetes: a systematic review. Lancet Diabetes Endocrinol. 2015;3:75-89.

32. Tuligenga RH. Intensive glycaemic control and cognitive decline in patients with type 2 diabetes: a meta-analysis. Endocr Connect. 2015;4:R16-24.

33. Aung PP, Strachan MW, Frier BM, Butcher I, Deary IJ, Price JF. Severe hypoglycaemia and late-life cognitive ability in older people with type 2 diabetes: the Edinburgh Type 2 Diabetes Study. Diabet Med. 2012;29:328-36.

34. Bruce DG, Davis WA, Casey GP, Clarnette RM, Brown SG, Jacobs IG, et al. Severe hypoglycaemia and cognitive impairment in older patients with diabetes: the Fremantle Diabetes Study. Diabetologia. 2009;52:1808-15.

35. de Galan BE, Zoungas S, Chalmers J, Anderson C, Dufouil C, Pillai A, et al. Cognitive function and risks of cardiovascular disease and hypoglycaemia in patients with type 2 diabetes: the Action in Diabetes and Vascular Disease: Preterax and Diamicron Modified Release Controlled Evaluation (ADVANCE) trial. Diabetologia. 2009;52:2328-36.

36. Punthakee Z, Miller ME, Launer LE, Williamson JD, Lazar RM, Cukierman-Yaffe T, et al. Poor cognitive function and risk of severe hypoglycemia in type 2 diabetes. Diabetes Care. 2012;35:787-93.

37. Yaffe K, Falvey CM, Hamilton N, Harris TB, Simonsick EM, Strotmeyer ES, et al. Association between hypoglycemia and dementia in a biracial cohort of older adults with diabetes mellitus. JAMA Intern Med. 2013;173:1300-6.

38. Feinkohl I, Aung PP, Keller M, Robertson CM, Morling JR, McLachlan S, et al. Severe hypoglycemia and cognitive decline in older people with type 2 diabetes: the Edinburgh Type 2 Diabetes Study. Diabetes Care. 2014;37:507-15.

39. Whitmer RA, Karter AJ, Yaffe K, Quesenberry CP, Selby JV. Hypoglycemic episodes and risk of dementia in older patients with type 2 diabetes. JAMA. 2009:301:1565-72.

40. Lin CH, Sheu WH. Hypoglycaemic episodes and risk of dementia in diabetes mellitus: 7-year follow-up study. J Intern Med. 2013;273:102-10.

41. Seaquist ER, Miller ME, Fonseca V, Ismail-Beigi F, Launer $L$, Punthakee Z, et al. Effect of thiazolidinediones and insulin on cognitive outcomes in ACCORD-MIND. J Diabetes Complications. 2013;27:485-91.

42. Misiak B, Leszek J, Kiejna A. Metabolic syndrome, mild cognitive impairment and Alzheimer's disease - the emerging role of systemic low-grade inflammation and adiposity. Brain Res Bull. 2012;89:144-9.

43. Marioni RE, Deary IJ, Murray GD, Lowe GDO, Strachan MW, Luciano M, et al. Genetic associations between fibrinogen and cognitive performance in three Scottish cohorts. Behav Genet. 2011;41:691-9.

44. Marioni RE, Strachan MW, Reynolds RM, Lowe GD, Mitchell RJ, Fowkes FG, et al. Association between raised inflammatory markers and cognitive decline in elderly people with type 2 diabetes: The Edinburgh Type 2 Diabetes Study. Diabetes Care. 2010;59:710-3.

45. Keller M, Feinkohl I, Anderson N, Deary IJ, Strachan MWJ, Price JF. Plasma fibrinogen and cognitive decline in older people with type 2 diabetes: the Edinburgh Type 2 Diabetes Study. Diabet Med. 2012;29:30-117.
46. Keller M, FeinkohI I, Anderson N, Deary IJ, Strachan MW, Price JF. Interleukin-6 predicts general cognitive decline and decline in non-verbal reasoning in older people with type 2 diabetes: the Edinburgh Type 2 Diabetes Study. Diabet Med. 2012;29:30-117.

47. Marioni RE, Deary IJ, Murray GD, Fowkes FG, Price JF. Associations between polymorphisms in five inflammation-related genes and cognitive ability in older persons. Genes Brain Behav. 2010;9:348-52.

48. Ding J, Patton N, Deary IJ, Strachan MW, Fowkes FG, Mitchell RJ, et al. Retinal microvascular abnormalities and cognitive dysfunction: a systematic review. Br J Ophthalmol. 2008;92:1017-25.

49. De Bresser J, Reijmer YD, van den Berg E, Breedijk MA, Kappelle L, Viergever MA, et al. Microvascular determinants of cognitive decline and brain volume change in elderly patients with type 2 diabetes. Dement Geriatr Cogn Disord. 2010;30:381-6.

50. Hugenschmidt CE, Lovato JF, Ambrosius WT, Bryan RN, Gerstein HC, Horowitz KR, et al. The cross-sectional and longitudinal associations of diabetic retinopathy with cognitive function and brain MRI findings: The Action to Control Cardiovascular Risk in Diabetes (ACCORD) Trial. Diabetes Care. 2014;37:3244-52.

51. Feinkohl I, Keller M, Robertson CM, Morling JR, Williamson RM, Nee LD, et al. Clinical and subclinical macrovascular disease as predictors of cognitive decline in older patients with type 2 diabetes: the Edinburgh Type 2 Diabetes Study. Diabetes Care. 2013;36:2779-86.

52. Daniels LB, Laughlin GA, Kritz-Silverstein D, Clopton P, Chen W-C, Maisel AS, et al. Elevated NT-proBNP levels are associated with poor cognitive function in community-dwelling older adults: results from the Rancho Bernardo Study. Am J Med. 2011;124:670.e1-9.e8.

53. Kerola T, Nieminen T, Hartikainen S, Sulkava R, Vuolteenaho O, Kettunen R. B-type natriuretic peptide as a predictor of declining cognitive function and dementia - a cohort study of an elderly general population with a 5-year follow-up. Ann Med. 2010;42:207-15.

54. Hiltunen M, Kerola T, Kettunen R, Hartikainen S, Sulkava R, Vuolteenaho O, et al. The prognostic capacity of B-type natriuretic peptide on cognitive disorders varies by age. Ann Med. 2013;45:74-8.

55. Vaes B, de Ruijter W, Degryse J, Westendorp RG, Gussekloo J. Clinical relevance of a raised plasma $\mathrm{N}$-terminal pro-brain natriuretic peptide level in a population-based cohort of nonagenarians. J Am Geriatr Soc. 2009;57:823-9.

56. Tynkkynen J, Laatikainen T, Salomaa V, Havulinna AS, Blankenberg S. NT-proBNP and the risk of dementia: a prospective cohort study with 14 years of follow-up. J Alzheimers Dis. 2015;44:1007-13.

57. Arntzen KA, Mathiesen EB. Subclinical carotid atherosclerosis and cognitive function. Acta Neurol Scand. 2011;124:18-22.

58. Trento M, Raballo M, Trevisan M, Sicuro J, Passera P, Cirio L, et al. A crosssectional survey of depression, anxiety, and cognitive function in patients with type 2 diabetes. Acta Diabetol. 2012;49:199-203.

59. Watari K, Letamendi A, Elderkin-Thompson V, Haroon E, Miller J, Darwin C, et al. A cross-sectional survey of depression, anxiety, and cognitive function in patients with type 2 diabetes. Arch Clin Neuropsychol. 2006;21:787-96.

60. Katon W, Lyles CR, Parker MM, Karter AJ, Huang ES, Whitmer RA. Association of depression with increased risk of dementia in patients with type 2 diabetes: The Diabetes and Aging Study. Arch Gen Psychiatry. 2012;69:410-7.

61. Sullivan MD, Katon WJ, Lavato LC, Miller ME, Murray AM, Horowitz KR, et al. Association of depression with accelerated cognitive decline among patients with type 2 diabetes in the ACCORD-MIND trial. JAMA Psychiatry. 2013;70:1041-7.

62. Mõttus R, Luciano M, Starr JM, Deary IJ. Diabetes and life-long cognitive ability. J Psychosom Res. 2013;75:275-8.

63. Bourne VJ, Fox HC, Deary IJ, Whalley LJ. Does childhood intelligence predict variation in cognitive change in later life? Pers Individ Dif. 2007:42:1551-9.

64. Gow AJ, Johnson W, Mishra G, Richards M, Kuh D, Deary IJ. Is age kinder to the initially more able? Yes, and no. Intelligence. 2012;40:49-59.

65. Götz J, Lim Y-A, Eckert A. Lessons from two prevalent amyloidoses - what amylin and $A \beta$ have in common. Front Aging Neurosci. 2013;5:1-10.

66. Craft S. The role of metabolic disorders in Alzheimer's disease and vascular dementia: two roads converged? Arch Neurol. 2009;66:300-5.

67. Takeda S, Sato N, Rakugi H, Morishita R. Molecular mechanisms linking diabetes mellitus and Alzheimer disease: beta-amyloid peptide, insulin signaling, and neuronal function. Mol Biosyst. 2011;7:1822-7.

68. Rojas A, Morales MA. Advanced glycation and endothelial functions: a link towards vascular complications in diabetes. Life Sci. 2004;76:715-30.

69. Jackson K, Barisone GA, Diaz E, Jin L-W, DeCarli C, Despa F. Amylin deposition in the brain: a second amyloid in Alzheimer's disease? Ann Neurol. 2013;74:517-26. 
70. Strachan MW, Reynolds RM, Frier BM, Mitchell RJ, Price JF. The relationship between type 2 diabetes and dementia. Br Med Bull. 2008;88:131-46.

71. Roberts RO, Knopman DS, Przybelski SA, Mielke MM, Kantarci K, Preboske GM, et al. Association of type 2 diabetes with brain atrophy and cognitive impairment. Neurology. 2014;82:1132-41.

72. Strachan MW. R D Lawrence Lecture 2010. The brain as a target organ in type 2 diabetes: exploring the links with cognitive impairment and dementia. Diabet Med. 2011;28:141-7. 